\title{
SPECTRAL SYNTHESIS FOR FLAT ORBITS IN THE DUAL SPACE OF WEIGHTED GROUP ALGEBRAS OF NILPOTENT LIE GROUPS
}

\author{
J. LUDWIG, C. MOLITOR-BRAUN, AND D. POGUNTKE
}

\begin{abstract}
Let $G=\exp (\mathfrak{g})$ be a connected, simply connected, nilpotent Lie group and let $\omega$ be a continuous symmetric weight on $G$ with polynomial growth. We determine the structure of all the two-sided closed ideals of the weighted group algebra $L_{\omega}^{1}(G)$ which are attached to a flat co-adjoint orbit.
\end{abstract}

\section{INTRODUCTION}

Let $A$ be a Banach algebra. In order to understand the structure of $A$ it is important to be able to determine the space of its two-sided closed ideals. A particular role is played by the primitive ideal space $\operatorname{Prim}(A)$ and its Fell topology. For a given closed subset $C$ of $\operatorname{Prim}(A)$, one tries to understand the set $\mathcal{I}^{C}$ of the two-sided closed ideals $I$ of $A$ with hull $C$. Here as always the hull $h(I)$ of $I$ is the closed subset $h(I)=\{J \in \operatorname{Prim}(A), I \subset J\}$ of $\operatorname{Prim}(A)$. We say that a closed subset $C$ of $\operatorname{Prim}(A)$ is spectral or of spectral synthesis if $\operatorname{ker}(C)=\bigcap_{J \in C} J$ is the only closed two-sided ideal of $A$ with hull $C$. We say that a two-sided closed ideal $I$ is primary if its hull consists of a single point in $\operatorname{Prim}(A)$. One important problem is, given an irreducible representation $\pi$ of $A$, to compute the space $\mathcal{I}^{\{\pi\}}$ of all the primary ideals with hull $\{\pi\}$.

In this paper we study the weighted $L^{1}$ algebra of a nilpotent simply connected connected Lie group $G=\exp (\mathfrak{g})$. For these groups the exponential mapping exp : $\mathfrak{g} \rightarrow G$ is a diffeomorphism of the vector space $\mathfrak{g}$ onto the group $G$ and the Haar measure $d g$ of $G$ is the pullback of Lebesgue measure on the vector space $\mathfrak{g}$. We say that a function $p: G \rightarrow \mathbb{C}$ is polynomial if the function $p \circ \exp : \mathfrak{g} \rightarrow \mathbb{C}$ is so. Let $\omega: G \rightarrow \mathbb{R}_{+}^{*}$ be a continuous symmetric polynomial weight, i.e. $\omega$ is a continuous strictly positive function defined on $G$, such that

$$
\omega(x)=\omega\left(x^{-1}\right), \omega(x y) \leq \omega(x) \omega(y), \omega(x) \geq C>0, \omega(x) \leq P(x), x, y \in G,
$$

where $C$ is a constant and $P: G \rightarrow \mathbb{R}$ is some polynomial function on $G$.

Received by the editors April 13, 2010 and, in revised form, February 1, 2012.

2010 Mathematics Subject Classification. Primary 22E30, 22E27, 43A20.

Key words and phrases. Nilpotent Lie group, irreducible representation, co-adjoint orbit, flat orbit, minimal ideal, spectral synthesis, weighted group algebra.

The second author was supported by the research grant 10NCHA of the University of Luxembourg. 
We consider the involutive Banach algebra $L_{\omega}^{1}(G)$ of integrable functions with respect to the measure $\omega d x$ on $G$. The product in this algebra is given by convolution,

$$
a * b(x)=\int_{G} a(u) b\left(u^{-1} x\right) d u, x \in G, a, b \in L_{\omega}^{1}(G),
$$

and its norm by

$$
\|a\|_{1, \omega}=\int_{G}|a(x)| \omega(x) d x, a \in L_{\omega}^{1}(G) .
$$

Then $\|a * b\|_{1, \omega} \leq\|a\|_{1, \omega}\|b\|_{1, \omega}$. This algebra has an isometric involution * given by

$$
a^{*}(x):=\overline{a\left(x^{-1}\right)}, x \in G, a \in L_{\omega}^{1}(G) .
$$

One fundamental question in non-commutative harmonic analysis consists in looking for relationships between geometric structures on one side and properties of representations, as well as ideals associated to them, on the other side. The most famous example in this respect is, of course, Kirillov's orbit theory (see Co-Gr] and [Le-Lu]) which gives us a homeomorphism $K: \hat{G} \rightarrow \mathfrak{g}^{*} / G$ from the dual space $\hat{G}$ of $G$ onto the space of co-adjoint orbits $\mathfrak{g}^{*} / G$ of $G$ in the real dual space $\mathfrak{g}^{*}$ of the Lie algebra $\mathfrak{g}$ of $G$.

The link with ideal theory of group algebras is the following: The primitive ideal space $\operatorname{Prim}\left(L_{\omega}^{1}(G)\right)$ of our algebra $L_{\omega}^{1}(G)$ is homeomorphic to the dual space $\hat{G}$ of $G$ and hence to the co-adjoint orbit space $\mathfrak{g}^{*} / G$ (see [Al-Lu] ). So, for a closed $G$-invariant subset $S$ of the linear dual $\mathfrak{g}^{*}$ of $\mathfrak{g}$, we shall now denote by $\mathcal{I}_{\omega}^{S}=\mathcal{I}^{S}$ the set of all closed two-sided ideals $I$ of $L_{\omega}^{1}(G)$, for which the hull $h(I):=\{\pi \in \widehat{G}, \pi(I)=\{0\}\}$ is the set of all irreducible unitary representations $\pi$ of $G$, whose Kirillov orbit $\Omega_{\pi}$ is contained in $S$.

A special property of the algebra $L_{\omega}^{1}(G)$ is the existence of minimal ideals, due to the fact that a connected nilpotent locally compact group has polynomial growth. This means that given any closed subset $C \subset \hat{G}$, there exists a minimal closed two-sided ideal $j(C) \subset L_{\omega}^{1}(G)$ with hull $C$, i.e. $j(C)$ is contained in every two-sided closed ideal $I$ with hull $h(I)$ equal to $C$ (see Dz-Lu-Mo ).

This naturally leads to the following fundamental problem: Given a polynomial weight on $G$ and an irreducible unitary representation $\pi$ of $G$, is it possible to deduce the properties of the algebra $\operatorname{ker}(\pi) / j(\{\pi\})$ from the geometric structure of the Kirillov orbit $\Omega_{\pi}=K(\pi)$ and the weight $\omega$ ? It has already been shown in [Lu1] that, for a flat orbit $\Omega_{\pi}$, the one-point set $\{\pi\}$ is spectral for the constant weight. But what about other weights?

As a matter of fact, non-trivial weights appear quite naturally in this type of problem. In the paper $\mathrm{Lu} 2$ the space of ideals $\mathcal{I}^{\{\pi\}}$ has been computed for every irreducible representation $\pi$ and any three step group $G$ again for the constant weight. It turned out that for every co-adjoint orbit $\Omega=K(\pi)$ in $\mathfrak{g}^{*}$ of such a group, there exists for $\ell \in \Omega$ a certain weight $\omega=\omega_{\Omega}$ on the stabilizer $G(\ell)=\exp (\mathfrak{g}(\ell))$ of $\ell$ such that the set $\mathcal{I}^{\{\pi\}}$ is in an inclusion preserving bijection with the set of ideals $\mathcal{I}_{\omega}^{\left\{\ell_{\mid \mathfrak{g}(\ell)}\right\}}$, and this set was easy to compute. Hence, in order to understand the fundamental algebra $L^{1}(G)$, one is led to study weighted group algebras, their representations and ideal theory, for more complicated weights, but easier groups and co-adjoint orbits. 
Another situation where weights occur is the following: In the course of the study of bounded topologically irreducible representations of the group algebras of exponential solvable Lie groups ([Lu-Mo-1], $[\mathrm{Po}]$ ), weighted group algebras of the type $L^{1}\left(\mathbb{R}^{n}, \omega\right)$ appear. Even though the group is just $\mathbb{R}^{n}$, the weight $\omega$ may be complicated and the non-unitary topologically irreducible representations of such algebras are not yet completely characterized. This fact stresses the importance of weighted group algebras in representation theory.

The problem of a link between the geometric structure of a co-adjoint orbit $\Omega_{\pi}$ and a weight $\omega$ with the algebra $\operatorname{ker}(\pi) / j(\{\pi\})$ is very complex and certainly constitutes an ambitious program. In the present paper we start this program by determining the sets $\mathcal{I}_{\omega}^{\Omega}$ for a flat co-adjoint orbit $\Omega$ and any polynomial weight $\omega$. An orbit is flat if it has the form $\Omega=\ell+\mathfrak{g}(\ell)^{\perp}$ for some $\ell \in \mathfrak{g}^{*}$. In Lu-Mo-2] the closed two-sided ideals in $\mathcal{I}_{\omega}^{\Omega}$ which are invariant by multiplication with functions from $L^{\infty}(G / G(l))$, where $G(l)=\exp (\mathfrak{g}(l))$, are completely determined. The purpose of this paper is to drop the $L^{\infty}(G / G(l))$-invariance condition and to describe all of $\mathcal{I}_{\omega}^{\Omega}$. It turns out that the space $\mathcal{I}_{\omega}^{\Omega}$ is not completely determined by the restriction of the weight $\omega$ to the stabilizer $G(\ell)$ of $\ell$. A member $I$ of $\mathcal{I}_{\omega}^{\Omega}$ is finitely generated modulo an $L^{\infty}(G / G(l))$-invariant ideal. See Theorem [5.5. Finally, in section 7 we show that the algebra $\mathcal{S}(G) / \mathcal{S}(G) \cap j\left(\pi_{l}\right)$ admits rank one projectors $\mathfrak{q}$ such that the two-sided, closed ideals of $\mathcal{S}(G) / \mathcal{S}(G) \cap j\left(\pi_{l}\right)$, and hence also of $L_{\omega}^{1}(G) / j\left(\pi_{l}\right)$, are in bijection with the set of two-sided ideals in the finitedimensional algebra $\mathfrak{q} * \mathcal{S}(G) / \mathcal{S}(G) \cap j\left(\pi_{l}\right) * \mathfrak{q}$. This is done via the theory of twisted convolution algebras. At the end of the paper we explicitly determine the set $\mathcal{I}_{\omega}^{\Omega}$ for the free nilpotent step two Lie algebra with 3 generators and a weight of degree one. For the 4-dimensional threadlike group, we determine one- or two-parameter families of ideals in $\mathcal{I}_{\omega}^{\Omega}$, if $\omega$ is of degree one or two.

To finish this introduction, let us just give examples of possible weights ([Lu-Mo-2] $)$. Let $\mathcal{P}$ be a finite-dimensional space of polynomial functions on $G$, which is invariant by left translations. Let $l_{\mathcal{P}}$ denote the representation of $G$ on $\mathcal{P}$ defined by

$$
\left(l_{\mathcal{P}}(x) P\right)(g):=P\left(x^{-1} g\right), \forall x, g \in G .
$$

Let $\|\cdot\|$ be any norm on $\mathcal{P}$ (as $\mathcal{P}$ is finite-dimensional, all the norms are equivalent). Then the symmetric weights $\omega$ and $\omega_{1}$ defined by

$$
\begin{aligned}
\omega(x) & :=\left\|l_{\mathcal{P}}(x)\right\|_{o p}+\left\|l_{\mathcal{P}}\left(x^{-1}\right)\right\|_{o p} \\
& =\sup _{P \in \mathcal{P}} \frac{\left\|l_{\mathcal{P}}(x) P\right\|}{\|P\|}+\sup _{P \in \mathcal{P}} \frac{\left\|l_{\mathcal{P}}\left(x^{-1}\right) P\right\|}{\|P\|}
\end{aligned}
$$

and

$$
\omega_{1}(x):=\max \left(\left\|l_{\mathcal{P}}(x)\right\|_{o p},\left\|l_{\mathcal{P}}\left(x^{-1}\right)\right\|_{o p}\right)
$$

have polynomial growth.

\section{Notation}

For a mapping $F: G \rightarrow E$ from $G$ into some set $E$, we denote by $l(g) F$ (resp. $r(g) F$, resp. $c(g) F)$ the left (resp. the right) translation (resp. the conjugation) of $F$ by $g \in G$, i.e. $l(g) F(u):=F\left(g^{-1} u\right), r(g) F(u):=F(u g), c(g) F(u):=$ $F\left(g^{-1} u g\right), u \in G$. 
We denote by $L_{\omega}^{\infty}(G)$ the Banach space of all measurable functions $\varphi: G \rightarrow \mathbb{C}$, for which $\|\varphi\|_{\infty, \omega}:=\operatorname{ess} \sup _{g \in G}\left|\frac{\varphi(g)}{\omega(g)}\right|<\infty$. This space $L_{\omega}^{\infty}(G)$ is in a natural way the Banach space dual of $L_{\omega}^{1}(G)$.

By $C(G)$ (resp. $C_{c}(G)$, resp. $C_{c}^{\infty}(G)$ ) we denote the space of all continuous functions (resp. continuous functions with compact support, resp. $C^{\infty}$-functions with compact support) $\varphi: G \rightarrow \mathbb{C}$. For an element $X \in \mathfrak{g}$ and for $\varphi \in C^{\infty}(G)$, define the left (resp. right) partial derivative $X * \varphi$ (resp. $\varphi * X)$ in the direction $X$ by

$$
X * \varphi(g):=\frac{d}{d t} \varphi(\exp (-t X) g)_{\mid t=0} \text { resp. } \varphi * X(g):=\frac{d}{d t} \varphi(g \exp (t X))_{\mid t=0}, g \in G .
$$

We extend the left and right partial derivatives in the usual way to the enveloping algebra $\mathcal{U}(\mathfrak{g})$ of $\mathfrak{g}$. For a closed $G$-left and right invariant subspace $W \subset L_{\omega}^{\infty}(G)$ we denote by $W^{\infty}$ the space consisting of all $C^{\infty}$-functions $\varphi: G \rightarrow \mathbb{C}$ contained in $W$, for which $a * \varphi * b \in W$ for all $a, b \in \mathcal{U}(\mathfrak{g})$. We denote the space $\left(L_{\omega}^{\infty}(G)\right)^{\infty}$ by the symbol $C_{\omega}^{\infty}(G)$.

Similarly, for a closed two-sided ideal $I$ of $L_{\omega}^{1}(G)$, we denote by $I^{\infty}$ the subspace of $I$ consisting of all the $C^{\infty}$ functions $f$ contained in $I$, for which $a * f * b \in I$ for all $a, b \in \mathcal{U}(\mathfrak{g})$.

Clearly, the subspace $I^{\infty}$ of $I$ (resp. $W^{\infty}$ of $W$ ) are $G$-left and right-invariant.

The following considerations can be found in Lu-Mo-2.

Let $N=\exp (\mathfrak{n})$ be a closed normal connected subgroup of $G$.

We say that the closed two-sided ideal $I \in \mathcal{I}^{\Omega}$ is $L^{\infty}(G / N)$-invariant if for every $f \in I$ and $\varphi \in L^{\infty}(G / N)$, the function $\varphi f$ is also in $I$.

We now consider the restriction $I_{\mid N}$ of a closed two-sided ideal in $L_{\omega}^{1}(G)$ to $N$, i.e., $I_{\mid N}$ is the closure in $L_{\omega_{\mid N}}^{1}(N)$ of the subspace generated by the functions $f * \beta_{\mid N}$, $\beta \in C_{c}(G), f \in I$. We recall that $I_{\mid N}$ is a closed two-sided $G$-invariant ideal of the algebra $L_{\omega_{\mid N}}^{1}(N)$. It is easy to see that for any dense subspace $I^{\prime}$ of $I$ we also have that

$$
I_{\mid N}=\overline{\operatorname{span}\left\{f * \alpha_{\mid N}, f \in I^{\prime}, \alpha \in C_{c}^{\infty}(G)\right\}} \|^{\|\|_{1, \omega} \mid N},
$$

where $\operatorname{span}\{\cdot\}$ denotes the vector subspace generated by $\{\cdot\}$. The converse construction is the extension $e_{G}(L)$ of a closed two-sided and $G$-invariant ideal $L \subset L_{\omega_{\mid N}}^{1}(N)$ to $G$. By definition,

$$
\begin{aligned}
e_{G}(L) & :={\overline{\operatorname{span}\left\{\beta * a, a \in L, \beta \in C_{c}(G)\right\}}}^{\|\|_{1, \omega}} \\
& ={\overline{\operatorname{span}\left\{a * \beta, a \in L, \beta \in C_{c}(G)\right\}}}^{\|\|_{1, \omega} .} .
\end{aligned}
$$

Then $e_{G}(L)$ is a closed two-sided ideal in $L_{\omega}^{1}(G)$. Furthermore it is the only one which is $L^{\infty}(G / N)$-invariant and whose restriction to $N$ equals $L$.

We denote by $\left(I^{\perp}\right)^{\infty}$ the functions $\varphi \in C_{\omega}^{\infty}(G)$, which are contained in the orthogonal space of $I$, i.e. for which

$$
\int_{G} f(g) \varphi(g) d g=0, f \in I .
$$

Also let $\left(I^{\perp}\right)_{\mid N}$ be the weak-* closure in $L_{\omega_{\mid N}}^{\infty}(N)$ of the vector space generated by the functions $\varphi_{\mid N}, \varphi \in\left(I^{\perp}\right)^{\infty}$.

An important tool in the proofs made in this paper will be the Schwartz algebra $\mathcal{S}(G)$ of $G$. 
Definition 2.1. The $S c h w a r t z$ algebra $\mathcal{S}(G)$ is the Fréchet sub-algebra of $L_{\omega}^{1}(G)$ consisting of all the $C^{\infty}$ functions $f: G \rightarrow \mathbb{C}$ for which all partial derivatives are rapidly decreasing, i.e. they are decreasing faster than the functions $\frac{1}{1+|P|}$ for any polynomial function $P$ on $G$ (see $[\mathrm{Lu}-\mathrm{Mo}$ for the properties of $S(G)$ ).

Remark 2.2. (i) Let $I_{\mid N}$ be as previously defined. We define $I_{N}$ by

$$
I_{N}:=\overline{\left.I \cap \mathcal{S}(G)\right|_{N}}\|\|_{1, \omega}{ }_{\mid N},
$$

where $\left.I \cap \mathcal{S}(G)\right|_{N}$ denotes the set of all the usual restrictions of the functions of $I \cap \mathcal{S}(G)$ to $N$. We then have $I_{N} \subset I_{\mid N}$. In fact, let $\left(\beta_{j}\right)_{j}$ be an approximate identity of the Schwartz algebra $\mathcal{S}(G)$, consisting of elements of $C_{c}^{\infty}(G)$ ([Lu-Mo $]$ ). Let $f \in I \cap \mathcal{S}(G)$. Then $f_{\mid N} \in \mathcal{S}(N)$ and $f * \beta_{j \mid N} \in I_{\mid N}$ for all $j$. Moreover, $\left(f * \beta_{j}\right)_{j}$ converges to $f$ in every Schwartz norm. This implies that $\left(f * \beta_{j \mid N}\right)_{j}$ converges to $f_{\mid N}$ in every Schwartz norm of $\mathcal{S}(N)$. As a matter of fact, if we build the Schwartz norms thanks to the supremum, restrictions to $N$ are possible. As the weight $\omega$ has polynomial growth, we then deduce that $\left(f * \beta_{j \mid N}\right)_{j}$ converges to $f_{\mid N}$ in $L_{\omega_{\mid N}}^{1}(N)$, and $f_{\mid N} \in I_{\mid N}$. This shows that

$$
I_{N} \subset I_{\mid N}
$$

(ii) We have

$$
I_{N}=I_{\mid N}, \text { provided } I=\overline{I \cap \mathcal{S}(G)}^{\|\|_{1, \omega}} .
$$

Indeed, $I_{\mid N}$ is then generated by elements of the form $f * \alpha_{\mid N}$ with $f \in I \cap \mathcal{S}(G)$ and $\alpha \in C_{c}^{\infty}(G)$. For such $f, \alpha, f * \alpha \in I \cap \mathcal{S}(G)$ and $f * \alpha_{\mid N} \in I_{N}$. This proves that $I_{\mid N} \subset I_{N}$.

(iii) If $I=\overline{I \cap \mathcal{S}(G)} \|^{\|\|_{1, \omega}}$, then

$$
I_{\mid N}=I_{N}=\overline{I_{N} \cap \mathcal{S}(N)} \|^{\|\|_{1, \omega}} .
$$

In fact $I_{N}$ is generated by the elements of $\left.I \cap \mathcal{S}(G)\right|_{N}$. But these are Schwartz functions on $N$.

(iv) Now let $M$ and $N$ be two closed connected normal subgroups of $G$ such that $N \subset M \subset G$ and let $I$ be a closed two-sided ideal in $L_{\omega}^{1}(G)$ such that $I=$ $\overline{I \cap \mathcal{S}(G)}^{\|\|_{1, \omega}}$. Then

$$
\left(I_{M}\right)_{N}=I_{N} \text { and }\left(\left(I^{\perp}\right)_{\mid M}\right)_{\mid N}=\left(I^{\perp}\right)_{\mid N} .
$$

In fact, $I_{M}$ is generated by $\left.I \cap \mathcal{S}(G)\right|_{M} \subset S(M)$ and so $\left(I_{M}\right)_{N}$ is generated by $\left.\left(\left(\left.I \cap \mathcal{S}(G)\right|_{M}\right) \cap \mathcal{S}(M)\right)\right|_{N}=\left.\left(\left.I \cap \mathcal{S}(G)\right|_{M}\right)\right|_{N}=\left.I \cap \mathcal{S}(G)\right|_{N}$, which shows that $\left(I_{M}\right)_{N}=I_{N}$. The fact that $\left(\left(I^{\perp}\right)_{\mid M}\right)_{\mid N}=\left(I^{\perp}\right)_{\mid N}$ is obvious from the definition of these spaces.

In order to study the relationship between $\left(I_{\mid N}\right)^{\perp}$ and $\left(I^{\perp}\right)_{\mid N}$, let us make the following decomposition of the Lie algebra $\mathfrak{g}$ : We take a subspace $\mathfrak{s} \subset \mathfrak{g}$ such that $\mathfrak{g}=\mathfrak{s} \oplus \mathfrak{n}$, such that the mapping

$$
\mathfrak{s} \times N \rightarrow G,(U, n) \mapsto \exp (U) n
$$

is a polynomial diffeomorphism and such that

$$
\int_{G} \varphi(g) d g=\int_{S} \int_{N} \varphi(s n) d n d s
$$


where $S=\exp (\mathfrak{s})$ and where $d s$ denotes Lebesgue measure on $S \simeq \mathfrak{s}$. Such subspaces $\mathfrak{s}$ always exist (see $[\mathrm{Co}-\mathrm{Gr}]$ ): In fact, if we take a strong Malcev basis $\mathcal{Z}=\left\{Z_{1}, \cdots, Z_{n}\right\}$ of the Lie algebra $\mathfrak{g}$ (i.e. the subspaces $\mathfrak{g}_{j}=\sum_{i=j}^{n} \mathbb{R} Z_{i}$ are ideals of $\mathfrak{g}(j=1, \cdots, n))$, which passes through $\mathfrak{n}$, i.e. $\mathfrak{n}=\mathfrak{g}_{m}$ for some $m$, then it suffices to take $\mathfrak{s}:=\sum_{j=1}^{m-1} \mathbb{R} Z_{j}$.

Proposition 2.3. $\quad$ (1) The subspace $\left(I_{\mid N}\right)^{\perp}$ is contained in $\left(I^{\perp}\right)_{\mid N}$.

(2) A two-sided closed ideal $I \subset L_{\omega}^{1}(G)$ is $L^{\infty}(G / N)$-invariant if and only if

$$
\left(I_{\mid N}\right)^{\perp}=\left(I^{\perp}\right)_{\mid N} .
$$

Proof. (1) Let $q \in\left(\left(I_{\mid N}\right)^{\perp}\right)^{\infty}$. Define a function $\varphi: G \rightarrow \mathbb{C}$ by the rule

$$
\varphi(s n):=\alpha(s) q(n), s \in S, n \in N,
$$

where $\alpha$ is an element of $C_{c}^{\infty}(S)$ such that $\alpha(e)=1$. Then $\varphi \in C_{\omega}^{\infty}(G)$ and $\varphi_{\mid N}=q$. Let us show that $\varphi \in I^{\perp}$. We have for $f \in I, \beta \in C_{c}(G)$,

$$
\begin{aligned}
\langle\varphi, f * \beta\rangle & =\int_{G} \varphi(g) f * \beta(g) d g \\
& =\int_{S} \alpha(s)\left(\int_{N} q(n) f * \beta(s n) d n\right) d s \\
& =\int_{S} 0 d s \\
& =0
\end{aligned}
$$

since the function $n \mapsto f * \beta(s n)$ is contained in $I_{\mid N}$ for every $s \in S$. Hence $\varphi \in\left(I^{\perp}\right)^{\infty}$ and $q \in\left(I^{\perp}\right)_{\mid N}$. Now if $q$ is any element of $\left(I_{\mid N}\right)^{\perp}$, then we can replace $q$ by functions of the type $\left.q_{\alpha, \beta}:=\alpha * q * \beta \in\left(I_{\mid N}\right)^{\perp}\right)^{\infty}$, with $\alpha, \beta \in$ $C_{c}^{\infty}(N)$. Since by the preceding argument we know that $q_{\alpha, \beta} \in\left(I^{\perp}\right)_{\mid N}$ for all $\alpha, \beta$, it follows that $q$ is contained in $\left(I^{\perp}\right)_{\mid N}$.

(2) If $I$ is $L^{\infty}(G / N)$-invariant, then for every $\varphi \in\left(I^{\perp}\right)^{\infty}$ and $\beta \in C_{c}^{\infty}(S)$ we have that

$$
\int_{S} \int_{N} f(s n) \beta(s) \varphi(s n) d n d s=0, f \in I * C_{c}(G) .
$$

Therefore $\int_{N} f(s n) \varphi(s n) d n=0, s \in S$, and so $\varphi_{\mid N} \in\left(I_{\mid N}\right)^{\perp}$. Hence $\left(I^{\perp}\right)_{\mid N}$ is contained in $\left(I_{\mid N}\right)^{\perp}$, and so by point (1) of this proposition $\left(I^{\perp}\right)_{\mid N}=\left(I_{\mid N}\right)^{\perp}$. If conversely $\left(I_{\mid N}\right)^{\perp}=\left(I^{\perp}\right)_{\mid N}$, then for $f \in I * C_{c}(G), \varphi \in$ $\left(I^{\perp}\right)^{\infty}, g \in G$, we have that

$$
l\left(g^{-1}\right) f \in I, l\left(g^{-1}\right) \varphi \in\left(I^{\perp}\right)^{\infty},
$$

and hence

$$
\int_{N} f(s n) \varphi(s n) d n=0, s \in S .
$$

We deduce from this that $\int_{S} \int_{N} \beta(s) f(s n) \varphi(s n) d n d s=0$ for any $\beta \in$ $C_{c}(G / N) \simeq C_{c}(S)$. This tells us that $\beta f \in I$. Since $C_{c}(G / N)$ is weak*dense in $L^{\infty}(G / N)$ and since $I * C_{c}(G)$ is norm dense in $I$, the assertion follows. 


\section{IdeAls WhOSE hUll is a flat orbit}

In this paper, we shall determine the space $\mathcal{I}^{\Omega}$ of all two-sided closed ideals in $L_{\omega}^{1}(G)$ with hull $\Omega$, where $\Omega$ is a flat co-adjoint orbit of $G$.

Let us recall that a co-adjoint orbit $\Omega=\operatorname{Ad}^{*}(G) \ell$ is flat if and only if the radical $\mathfrak{g}(\ell):=\{U \in \mathfrak{g},\langle\ell,[U, \mathfrak{g}]=\{0\}\rangle\}$ of $\ell$ is an ideal in $\mathfrak{g}$ and if and only if $\Omega=\ell+\mathfrak{g}(\ell)^{\perp}$ (see $[\mathrm{Lu}]$ ).

In the sequel we shall fix the element $\ell$ whose orbit is flat and write $\mathfrak{n}=\mathfrak{g}(\ell), N=$ $\exp (\mathfrak{n})$, which is a closed connected normal subgroup of $G$. Let $p=\ell_{\mid \mathfrak{n}}$, which is $G$-invariant as $\mathfrak{n}$ is the stabilizer of $l$ in $\mathfrak{g}$, which is an ideal by assumption. The function

$$
\chi_{p}=\chi_{\ell_{\mid \mathfrak{n}}}: N \rightarrow \mathbb{C}, \chi_{\ell_{\mid \mathfrak{n}}}(n):=e^{-i \ell(\log (n))}, n \in N,
$$

defines a unitary character of $N$, which is hence also $G$-invariant, i.e. $\chi_{p}\left(g n g^{-1}\right)=$ $\chi_{p}(n)$ for every $g \in G, n \in N$.

In the case where $\ell$ is a homomorphism of $\mathfrak{g}$, the space $\mathcal{I}^{\{\ell\}}$ has been determined in $\mathrm{Al}-\mathrm{Lu}$ : We denote by $\mathcal{P}_{\omega}(G)$ the complex finite-dimensional vector space of all polynomial functions $P: G \rightarrow \mathbb{C}$ contained in $L_{\omega}^{\infty}(G)$ and by $\mathcal{P} \mathcal{I}_{\omega}(G)$ the set of all left and right translation invariant subspaces of $\mathcal{P}_{\omega}(G)$. For every $V \in \mathcal{P} \mathcal{I}_{\omega}(G)$, let

$$
J_{V}:=\left\{f \in L_{\omega}^{1}(G), \int_{G} f(g) P(g) \chi_{\ell}(g) d g=0, \forall P \in V\right\} .
$$

Then $J_{V}$ is a closed two-sided ideal of the algebra $L_{\omega}^{1}(G)$ and the mapping $\mathcal{P} \mathcal{I}_{\omega}(G) \ni$ $V \mapsto J_{V} \in \mathcal{I}^{\{\ell\}}$ is an order reversing bijection.

If $\ell$ is not a homomorphism, i.e. if the $G$-orbit $\Omega$ of $\ell$ is not reduced to a single point in $\mathfrak{g}^{*}$, the problem of determining the space $\mathcal{I}^{\Omega}$ is very difficult (see for instance $\mathrm{Lu} 2$, where $\mathcal{I}^{\Omega}$ is determined for $\omega=1$ and $\mathfrak{g}$ of step 3 ). In this paper we consider the case of flat orbits. Then for the trivial weight $\omega=1$, the orbit $\Omega \in \mathfrak{g}^{*} / G$ is of spectral synthesis (see [Lu1]).

Definition 3.1. Let $\mathcal{P}_{\omega_{\mid N}}(N)$ be the complex vector space of all polynomial functions $P: N \rightarrow \mathbb{C}$, which are dominated by $\omega_{\mid N}$.

Let $\mathcal{P} \mathcal{I}_{\omega_{\mid N}}(N)^{G}$ be the space of $G$-conjugation invariant and $N$ translationinvariant subspaces of $\mathcal{P}_{\omega_{\mid N}}(N)$. For every $V \in \mathcal{P I}_{\omega_{\mid N}}(N)^{G}$, we define $J_{V} \subset$ $L_{\omega_{\mid N}}^{1}(N)$ as previously.

The following theorem proved in Lu-Mo-2] determines the space of $L^{\infty}(G / N)$ invariant ideals in $\mathcal{I}^{\Omega}$ for a flat co-adjoint orbit $\Omega \subset \mathfrak{g}^{*}$.

Theorem 3.2. Suppose that $\Omega$ is a flat co-adjoint orbit. The mapping

$$
\mathcal{P} \mathcal{I}_{\omega_{\mid N}}(N)^{G} \rightarrow \mathcal{I}^{\Omega}, V \mapsto I_{V}:=e_{G}\left(J_{V}\right)
$$

is order reversing and a bijection between $\mathcal{P} \mathcal{I}_{\omega_{\mid N}}(N)^{G}$ and the space of $L^{\infty}(G / N)$ invariant ideals contained in $\mathcal{I}^{\Omega}$.

Remark 3.3. Since $I_{V}$ is an $L^{\infty}(G / N)$-invariant ideal, it follows that

$$
I_{V}=\left\{f \in L_{\omega}^{1}(G), \int_{S} \int_{N} \varphi(s) p(n) \chi_{l}(n) f(s n) d n d s=0, \varphi \in C_{c}(G / N), p \in V\right\} .
$$

In this paper we prove a structure theorem for every two-sided closed ideal $I \subset L_{\omega}^{1}(G)$ with hull $\Omega$; see Theorem 5.5 . 
Definition 3.4. For $P \in \mathcal{P}_{\omega \mid N}(N)$ write

$$
l(m) P(n):=P\left(m^{-1} n\right), r(m) P(n):=P(n m), \sigma(g) P(n):=P\left(g^{-1} n g\right),
$$

$m, n \in N, g \in G$, for left and right translation by $m \in N$ and conjugation by $g \in G$. For $Z \in N, X \in \mathfrak{g}$, we have that

$$
l(\exp (Z))=\sum_{j=0}^{d} \frac{d l(Z)^{j}}{j !}, r(\exp (Z))=\sum_{j=0}^{d} \frac{d r(Z)^{j}}{j !},
$$

and

$$
\sigma(\exp (X))=\sum_{j=0}^{d} \frac{d \sigma(X)^{j}}{j !}, X \in \mathfrak{g},
$$

where $d=\operatorname{dim}\left(\mathcal{P}_{\omega_{\mid N}}(N)\right)$, since $r, l$ and $\sigma$ are unipotent representations of $N$ (resp. $G)$ on $\mathcal{P}_{\omega_{\mid N}}(N)$.

It had been shown in Lu-Mo-2 that the minimal ideal $j(\Omega)=j\left(\pi_{\ell}\right)$ is the extension of the minimal ideal $j\left(\chi_{p}\right) \subset L_{\omega_{\mid N}}^{1}(N)$.

Since

$$
\begin{aligned}
j(\{p\})=j\left(\chi_{p}\right) & =\left\{f \in L_{\omega_{\mid N}}^{1}(N), \int_{N} f(n) \chi_{p}(n) P(n) d n=0, \forall P \in \mathcal{P}_{\omega_{\mid N}}(N)\right\} \\
& =J_{\mathcal{P}_{\omega_{\mid N}}(N)}
\end{aligned}
$$

by $[\mathrm{Al}-\mathrm{Lu}]$, the minimal ideal $j(\Omega)$ being the extension to $G$ of $j\left(\chi_{p}\right)$, we have that $(3.2)$

$j(\Omega)=j\left(\pi_{\ell}\right)=\left\{f \in L_{\omega}^{1}(G), \int_{N} f * \beta(n) P(n) \chi_{p}(n) d n=0, \beta \in C_{c}(G), P \in \mathcal{P}_{\omega_{\mid N}}(N)\right\}$.

Remark 3.5. Let $d$ be the dimension of the vector space $L_{\omega_{\mid N}}^{1}(N) / j\left(\chi_{p}\right)$, i.e. $d=$ $\operatorname{dim}\left(\mathcal{P}_{\omega_{\mid N}}\right)$. Then $\left(\operatorname{ker}\left(\pi_{l}\right)\right)^{d} \subset j\left(\chi_{p}\right)$.

Indeed, by Lu-Mo-2, we know that $\overline{\operatorname{ker}(\pi)^{j}}$ is the extension of $\left(\operatorname{ker}\left(\chi_{p}\right)\right)^{j}, j=$ $1,2, \cdots$. But since $\operatorname{ker}\left(\chi_{p}\right) / j\left(\chi_{p}\right)$ is nilpotent, it follows that $\operatorname{ker}\left(\chi_{p}\right)^{d} \subset j\left(\chi_{p}\right)$. Hence $\left(\operatorname{ker}\left(\pi_{\ell}\right)\right)^{d} \subset e\left(\operatorname{ker}\left(\chi_{p}\right)^{d}\right) \subset e\left(j\left(\chi_{p}\right)\right)=j\left(\pi_{l}\right)$.

Now if $I \in \mathcal{I}^{\Omega}$, then $I$ contains the minimal ideal $j(\Omega)$. Therefore, for any $\varphi \in\left(I^{\perp}\right)^{\infty}, g \in G$, the function

$$
\varphi(g): N \rightarrow \mathbb{C}, n \mapsto \varphi(g n)
$$

is contained in $j\left(\chi_{p}\right)^{\perp}=\chi_{p} \mathcal{P}_{\omega_{\mid N}}(N)$. Hence the functions $\tilde{\varphi}(g), g \in G$, defined by

$$
\tilde{\varphi}(g)(n)=\overline{\chi_{p}(n)} \varphi(g n), n \in N,
$$

are polynomial and bounded by $\omega_{\mid N}(N)$, if $\varphi \in\left(I^{\perp}\right)^{\infty}$, by Lu-Mo-2.

The set of all these polynomial functions $\tilde{\varphi}(g), g \in G, \varphi \in\left(I^{\perp}\right)^{\infty}$, generates a $G \times N$-invariant subspace of $\mathcal{P}_{\omega_{\mid N}}(N)$, which we denote by $V(I)$. This means that $\left(I^{\perp}\right)_{\mid N}=\chi_{p} V(I)$.

Let $I$ be a closed two-sided ideal of $L_{\omega}^{1}(G)$ with hull $\Omega$. Hence $j\left(\pi_{l}\right) \subset I$ and $I^{\perp} \subset j\left(\pi_{l}\right)^{\perp}$. As $j\left(\pi_{l}\right)$ is $L^{\infty}(G / N)$-invariant, by Lu-Mo-2], Proposition 2.3 implies that

$$
\left(j\left(\pi_{l}\right)^{\perp}\right)_{\mid N}=\left(j\left(\pi_{l}\right)_{\mid N}\right)^{\perp}=\left(j\left(\chi_{l_{\mid \mathfrak{n}}}\right)\right)^{\perp} .
$$


Hence

$$
\begin{aligned}
W(I) & :=\overline{\chi_{p}} \cdot\left(I_{\mid N}\right)^{\perp} \\
& \subset \\
V(I) & :=\overline{\chi_{p}} \cdot\left(I^{\perp}\right)_{\mid N} \\
& \subset \\
\overline{\chi_{p}} \cdot\left(j\left(\chi_{p}\right)\right)^{\perp} & =\mathcal{P}_{\omega_{\mid N}}(N) .
\end{aligned}
$$

Remark 3.6. By definition of $V(I)$ and $W(I)$,

$$
I_{V(I)} \subset I \subset I_{W(I)}
$$

and $W(I)=V(I)$ if and only if $I$ is $L^{\infty}(G / N)$-invariant, by Proposition 2.3, If $I$ is $L^{\infty}(G / N)$-invariant, then $I=I_{V(I)}=I_{W(I)}$, by Theorem 3.2 This will be specified further in Theorem 5.5 .

Moreover, $I_{V(I)}$ is the largest $L^{\infty}(G / N)$-invariant ideal contained in $I$ with hull $\Omega$ and $I_{W(I)}$ is the smallest $L^{\infty}(G / N)$-invariant ideal containing $I$ with hull $\Omega$.

In fact, let us assume that $\tilde{I}$ is an $L^{\infty}(G / N)$-invariant ideal with hull $\Omega$ such that $\tilde{I} \subset I$. Then $V(I) \subset V(\tilde{I})$ and $\tilde{I}=I_{V(\tilde{I})} \subset I_{V(I)}$, which completes the proof of the first statement. The second one is proved similarly.

Remark 3.7. Let $\left(p_{j}\right)_{j=1}^{d}$ be a basis of the (finite-dimensional) vector space $\mathcal{P}_{\omega_{\mid N}}(N)$. Let us take this basis to be a Jordan-Hölder basis for the (unipotent) action of $G \times N$ on $\mathcal{P}_{\omega_{\mid N}}(N)$. For a function $\varphi \in\left(j(\Omega)^{\perp}\right)^{\infty}$ we can express the map $\tilde{\varphi}$ in the basis $P$ :

$$
\chi_{p}(n) \tilde{\varphi}(g)(n)=\varphi(g n)=\sum_{j=1}^{d} \varphi_{j}(g) p_{j}(n) \chi_{p}(n), g \in G, n \in N .
$$

It is obvious that the functions $\varphi_{j}$ are $C^{\infty}$.

Lemma 3.8. The complex functions $\varphi_{j}$ are then contained in $C_{\omega}^{\infty}(G)$.

Proof. Indeed, choose a family of Schwartz functions $\left(\delta_{j}\right)_{j=1}^{d} \subset \mathcal{S}(N)$ such that

$$
\int_{N} p_{j}(n) \chi_{p}(n) \delta_{i}(n) d n=\delta_{i j}, i, j=1, \cdots, d .
$$

Then

$$
\begin{aligned}
\left|\varphi_{j}(g)\right| & =\left|\int_{N} \tilde{\varphi}(g)(n) \chi_{p}(n) \delta_{j}(n) d n\right| \\
& \leq \int_{N}|\tilde{\varphi}(g)(n)|\left|\delta_{j}(n)\right| d n \\
& =\int_{N}\left|\varphi(g n) \| \delta_{j}(n)\right| d n \\
& \leq\|\varphi\|_{\infty, \omega} \int_{N} \omega(g) \omega(n)\left|\delta_{j}(n)\right| d n \\
& \leq\|\varphi\|_{\infty, \omega}\left\|\delta_{j}\right\|_{1, \omega \mid N} \omega(g) .
\end{aligned}
$$

Hence

$$
\left\|\varphi_{j}\right\|_{\infty, \omega} \leq\|\varphi\|_{\infty, \omega}\left\|\delta_{j}\right\|_{1, \omega_{\mid N}} .
$$


We have to prove similar bounds for $u * \varphi * v, u, v \in \mathcal{U}(\mathfrak{g})$. Let us first notice that ${ }_{y} \varphi, \varphi_{y} \in\left(j(\Omega)^{\perp}\right)^{\infty}$ for all $y \in G$, where ${ }_{y} \varphi(g):=\varphi\left(y^{-1} g\right)$ and $\varphi_{y}(g)=\varphi(g y)$. We then have

$$
\widetilde{\left({ }_{y} \varphi\right)}(g)(n)=\tilde{\varphi}\left(y^{-1} g\right)(n)=\sum_{j=1}^{d} \varphi_{j}\left(y^{-1} g\right) p_{j}(n)=\sum_{j=1}^{d}{ }_{y}\left(\varphi_{j}\right)(g) p_{j}(n),
$$

and hence

$$
\left({ }_{y} \varphi\right)_{j}={ }_{y}\left(\varphi_{j}\right) \text {, for all } j
$$

This implies that

$$
(X * \varphi)_{j}=X *\left(\varphi_{j}\right), X \in \mathfrak{g}
$$

and even

$$
(u * \varphi)_{j}=u *\left(\varphi_{j}\right), u \in \mathcal{U}(\mathfrak{g}) .
$$

Hence for any $u \in \mathcal{U}(\mathfrak{g})$ the function $u *\left(\varphi_{j}\right)$ is contained in $L_{\omega}^{\infty}(G)$ as

$$
\begin{aligned}
\left\|X *\left(\varphi_{j}\right)\right\|_{\infty, \omega} & =\left\|(X * \varphi)_{j}\right\|_{\infty, \omega} \\
& \leq\|X * \varphi\|_{\infty, \omega}\left\|\delta_{j}\right\|_{1, \omega_{\mid N}} \\
& <+\infty
\end{aligned}
$$

We have similar bounds if $X$ is replaced by $u \in \mathcal{U}(\mathfrak{g})$ because of the assumptions on $\varphi$.

Before dealing with the action of $X$ on the right, let us notice the following: As $\mathcal{P}_{\omega \mid N}(N)$ is $G$-invariant and admits $\left(p_{k}\right)$ as a basis,

$$
p_{j}\left(y^{-1} n y\right)=\sum_{k=1}^{d} \rho_{j k}(y) p_{k}(n), \forall y \in G, \forall n \in N,
$$

where the $\rho_{j k}$ 's are polynomial functions. For the unit $y=e$ one has $\rho_{j k}(e)=\delta_{j k}$ because of the unicity of the decomposition in the basis $\left(p_{k}\right)_{k=1}^{d}$. For $y=\exp (t X)$, we put $r_{j k}(t):=\rho_{j k}(\exp (t X))$. Then the $r_{j k}$ 's are polynomial functions in $t$ such that $r_{j k}(0)=\rho_{j k}(e)=\delta_{j k}$. One easily checks that for $g \in G$,

$$
\left(\varphi_{\exp (t X)}\right)_{j}(g)=\sum_{i=1}^{d} r_{i j}(t)\left(\varphi_{i}\right)_{\exp (t X)}(g)
$$

and hence

$$
\begin{aligned}
\sum_{j=1}^{d}(\varphi * X)_{j}(g) p_{j}(n) & =\overline{\chi_{p}(n)} \cdot(\varphi * X)(g n) \\
& =\sum_{j=1}^{d}\left(\sum_{i=1}^{d} r_{i j}^{\prime}(0) \cdot \varphi_{i}(g)+\sum_{i=1}^{d} r_{i j}(0) \cdot\left(\varphi_{i} * X\right)(g)\right) p_{j}(n) .
\end{aligned}
$$

So

$$
\begin{aligned}
(\varphi * X)_{j}(g) & =\sum_{i=1}^{d}\left(r_{i j}^{\prime}(0) \cdot \varphi_{i}(g)+r_{i j}(0)\left(\varphi_{i} * X\right)(g)\right) \\
& =\sum_{i=1}^{d} r_{i j}^{\prime}(0) \cdot \varphi_{i}(g)+\left(\varphi_{j} * X\right)(g)
\end{aligned}
$$


and

$$
\left(\varphi_{j} * X\right)(g)=(\varphi * X)_{j}(g)-\sum_{i=1}^{d} r_{i j}^{\prime}(0) \varphi_{i}(g) .
$$

The previous bounds imply that

$$
\begin{aligned}
\left\|\varphi_{j} * X\right\|_{\infty, \omega} & \leq\left\|(\varphi * X)_{j}\right\|_{\infty, \omega}+\sum_{i=1}^{d}\left|r_{i j}^{\prime}(0)\right|\left\|\varphi_{i}\right\|_{\infty, \omega} \\
& \leq\|\varphi * X\|_{\infty, \omega}\left\|\delta_{j}\right\|_{1, \omega \mid N}+\sum_{i=1}^{d}\left|r_{i j}^{\prime}(0)\right|\|\varphi\|_{\infty, \omega}\left\|\delta_{i}\right\|_{1, \omega_{\mid N}} .
\end{aligned}
$$

For any $u, v \in \mathcal{U}(\mathfrak{g})$, we then get a bound of the form

$$
\begin{aligned}
\left\|u * \varphi_{j} * v\right\|_{\infty, \omega} & =\left\|(u * \varphi)_{j} * v\right\|_{\infty, \omega} \\
& \leq \sum_{i=1}^{d}\left(c_{i}\|u * \varphi\|_{\infty, \omega}+\sum_{k=1}^{N_{i}}\left\|u * \varphi * v_{i k}\right\|_{\infty, \omega}\right)\left\|\delta_{i}\right\|_{1, \omega_{\mid N}} \\
& <+\infty
\end{aligned}
$$

by assumption on $\varphi$, for some $v_{i k} \in \mathcal{U}(\mathfrak{g})$ and some non-negative constants $c_{i}$. This proves that $\varphi_{j} \in C_{\omega}^{\infty}(G)$, for all $j$.

Definition 3.9. For any closed two-sided ideal $I \subset L_{\omega}^{1}(G)$, we let

$$
I^{S}=I \cap \mathcal{S}(G)
$$

be the intersection of $I$ with $\mathcal{S}(G)$.

Remark 3.10. It follows from $[\mathrm{Lu} 2$ and $\mathrm{Lu}-\mathrm{Mo}$ that the hulls of $I$ and of $\overline{I \cap \mathcal{S}(G)}^{L_{\omega}^{1}(G)}$ coincide. As a matter of fact, the minimal ideal $j(h(I))$ is generated by Schwartz functions. Hence $h(I \cap \mathcal{S}(G))=h(I)$.

The next theorem will be an important step in the proof of Theorem 5.5

Theorem 3.11. Let $\Omega$ be a flat $G$-orbit in $\mathfrak{g}^{*}$. Let $I \in \mathcal{I}^{\Omega}$ be a closed two-sided ideal in $L_{\omega}^{1}(G)$. Then $I=\overline{I^{S}}$, the closure being taken in $L_{\omega}^{1}(G)$. Moreover, every $f \in I^{\infty}$ is in $\mathcal{S}(G)$ modulo $j(\Omega)$.

In order to prove Theorem 3.11 we first establish the following lemma:

Lemma 3.12. Let $\left(p_{j}\right)_{j=1}^{d}$ be a Jordan-Hölder basis of $\mathcal{P}_{\omega_{\mid N}}(N)=\overline{\chi_{p}} \cdot j\left(\chi_{p}\right)^{\perp}$ for the action of $G \times N$, where $d=\operatorname{dim}\left(j\left(\chi_{p}\right)^{\perp}\right)$. We extend the functions $p_{j}$ to polynomial functions defined on all of $G$ by letting

$$
P_{j}(s n):=p_{j}(n), n \in N, s \in S .
$$

Then there exists a function $q \in C_{\omega}^{\infty}(S) \equiv C_{\omega}^{\infty}(G / N)$ such that $0<q \omega \leq C$ for some constant $C>0$ and such that all the right and left partial derivatives of the functions

$$
r_{j}:=q P_{j}, j=1, \ldots, d
$$

are dominated by $\omega$. 
Proof. Let $\omega^{\prime}$ be a symmetric continuous polynomial weight on $S \equiv G / N$ such that

$$
\omega^{\prime}(s) \geq C_{2} \omega^{2}(s) \geq C_{1}>0, s \in S,
$$

for some strictly positive constants $C_{1}, C_{2}$. Such a weight exists as $G / N$ is a locally compact group of polynomial growth on which every polynomially bounded function is bounded by a polynomial weight ([Di], Lu4]). We define $\omega^{\prime}$ on all of $G$ by $\omega^{\prime}(s n):=\omega^{\prime}(s)$, for all $s \in S, n \in N$. For any non-negative functions $\alpha, \beta \in C_{c}(G)$, we have

$$
\begin{aligned}
\alpha * \omega^{\prime} * \beta(x) & =\int_{G} \int_{G} \alpha(y) \omega^{\prime}\left(y^{-1} x u^{-1}\right) \beta(u) d u d y \\
& \leq \omega^{\prime}(x)\left(\int_{G} \alpha(y) \omega^{\prime}(y) d y\right)\left(\int_{G} \beta(u) \omega^{\prime}(u) d u\right) .
\end{aligned}
$$

Now let $\alpha \in C_{c}^{\infty}(G), \alpha \geq 0$, such that $\int_{G} \alpha(g) \omega^{\prime}(g) d g=1$. We define a $C^{\infty}$ function $\mu$ by $\mu:=\alpha * \omega^{\prime} * \alpha$ and we put $q=\frac{1}{\mu}$. Hence

$$
\begin{aligned}
\left(\int_{G} \frac{\alpha(y)}{\omega^{\prime}(y)} d y\right)^{2} \cdot \omega^{\prime}(x) & \leq \int_{G} \int_{G} \alpha(y) \alpha(u) \omega^{\prime}\left(y^{-1} x u^{-1}\right) d y d u \\
& =\mu(x) \\
& \leq \omega^{\prime}(x)
\end{aligned}
$$

for all $x \in G$, i.e. there exist strictly positive constants $C_{3}, C_{4}$ such that

$$
0<C_{3} \leq C_{4} \cdot \omega^{\prime}(g) \leq \mu(g) \leq \omega^{\prime}(g), \forall g \in G .
$$

For $j=1, \cdots, d$, we have

$$
\begin{aligned}
\left|P_{j}(s n)\right| & =\left|p_{j}(n)\right| \\
& \leq C_{5} \omega(n) \\
& \leq C_{5} \omega(s) \omega(s n) \\
& \leq C_{6} \omega(s)^{2} \omega(s n) \\
& \leq C_{7} \omega^{\prime}(s) \omega(s n) \\
& =C_{7} \omega^{\prime}(s n) \omega(s n)
\end{aligned}
$$

for some strictly positive constants $C_{5}, C_{6}, C_{7}$. Hence $P_{j} \in \mathcal{P}_{\omega^{\prime} \omega}(G)$. Similarly for $u * P_{j}$, for all $u \in \mathcal{U}(\mathfrak{g})$, for all $j$, with the constants depending on $u$. For all $u \in \mathcal{U}(\mathfrak{g})$, we have

$$
|u * \mu|=\left|(u * \alpha) * \omega^{\prime} * \alpha\right| \leq|u * \alpha| * \omega^{\prime} *|\alpha| \leq C_{8} \cdot \omega^{\prime} \leq C_{9} \cdot \mu
$$

for new constants $C_{8}, C_{9}$ (depending on $u$ ). For $X \in \mathfrak{g}$ and $j \in\{1, \cdots, d\}$,

$$
\begin{aligned}
\left|X * r_{j}\right| & =\left|X *\left(P_{j} q\right)\right| \\
& =\left|\left(X * P_{j}\right) q+P_{j}(X * q)\right| \\
& \leq C_{10} \omega \omega^{\prime} q+\left|P_{j}\left(X * \frac{1}{\mu}\right)\right| \\
& \leq C_{10} \omega \omega^{\prime} \frac{1}{\mu}+C_{10} \omega \omega^{\prime} \frac{|X * \mu|}{\mu^{2}} \\
& \leq C_{10} \omega \frac{\omega^{\prime}}{\mu}+C_{10} \omega \omega^{\prime} \frac{C_{9} \mu}{\mu^{2}} \\
& \leq C_{11} \omega
\end{aligned}
$$


for new constants $C_{10}, C_{11}$. Hence $X * r_{j}$ is dominated by $\omega$. Similarly for $r_{j} * X$. The same is true for derivatives of higher order. One also shows that all the right and left derivatives of $q$ are bounded by $\omega$. Finally,

$$
\begin{aligned}
q \omega(s) & =q(s) \omega(s) \\
& =\frac{\omega(s)}{\mu(s)} \\
& \leq \frac{C_{12} \omega^{2}(s)}{\mu(s)} \\
& \leq \frac{C_{13} \omega^{\prime}(s)}{\mu(s)} \\
& \leq C
\end{aligned}
$$

for new constants $C_{12}, C_{13}, C$. It is clear that the function $\frac{1}{q}=\mu$ has the desired properties.

Remark 3.13. (i) Let $l \in \mathfrak{g}^{*}, \mathfrak{p}(l)$ be a polarization at the point $l, P(l)=\exp (l)$, $\pi_{l}=\operatorname{ind}_{P(l)}^{G} \chi_{l}$ the corresponding induced representation, $\mathcal{H}_{l}$ the Hilbert space of the representation $\pi_{l}$ and $\mathcal{B}\left(\mathcal{H}_{l}\right)$ the Banach space of all bounded endomorphisms of $\mathcal{H}_{l}$. Then $\mathcal{B}\left(\mathcal{H}_{l}\right)$ is a $G \times G$-module, the action of $G \times G$ being given by

$$
\left(g, g^{\prime}\right) a:=\pi_{l}(g) \circ a \circ \pi_{l}\left(\left(g^{\prime}\right)^{-1}\right), a \in \mathcal{B}\left(\mathcal{H}_{l}\right), g, g^{\prime} \in G .
$$

Let $\mathcal{B}\left(\mathcal{H}_{l}\right)^{\infty}$ denote the space of $C^{\infty}$-vectors for this action. Then

$$
\pi_{l}\left(L^{1}(G)^{\infty}\right) \subset \mathcal{B}\left(\mathcal{H}_{l}\right)^{\infty}
$$

In fact, for all $u \in \mathcal{U}(\mathfrak{g})$, the operator $d \pi_{l}(u)$ acts on the representation space as a differential operator with polynomial coefficients, and conversely. This means that for all differential operators with polynomial coefficients $P, Q$ on the representation space $\mathcal{H}_{l}^{\infty}$ (identified with a space $S\left(\mathbb{R}^{s}\right)$ ), there are $u, v \in \mathcal{U}(\mathfrak{g})$ such that $P=$ $d \pi_{l}(u), Q=d \pi_{l}(v)$ and

$$
\begin{aligned}
\left\|P \circ \pi_{l}(f) \circ Q\right\|_{o p} & =\left\|\pi_{l}(u * f * v)\right\|_{o p} \\
& \leq\|u * f * v\|_{L^{1}(G)} \\
& <+\infty
\end{aligned}
$$

for all $f \in L^{1}(G)^{\infty}$.

(ii) By $[\mathrm{Ho}$, the map

$$
\begin{array}{cc}
\mathcal{S}(G) & \rightarrow \mathcal{B}\left(\mathcal{H}_{l}\right)^{\infty} \\
f & \mapsto \pi_{l}(f)
\end{array}
$$

is surjective. So, for every $k \in L^{1}(G)^{\infty}$, there exists $f \in \mathcal{S}(G)$ such that $\pi_{l}(k)=$ $\pi_{l}(f)$.

Proof of Theorem 3.11. We define a mapping $\Phi: L_{\omega}^{1}(G) \rightarrow\left(\mathcal{B}\left(\mathcal{H}_{\ell}\right)\right)^{d}$ by the rule

$$
\Phi(f):=\left(\pi_{\ell}\left(r_{j} f\right)\right)_{j=1}^{d}, f \in L_{\omega}^{1}(G) .
$$


Then, since $\operatorname{ker}\left(\pi_{\ell}\right)=\operatorname{ker}\left(\operatorname{ind}_{N}^{G} \chi_{p}\right)$ (see [Lu-Mo-2]), it follows that

$$
\begin{aligned}
& \operatorname{ker}(\Phi)=\left\{f \in L_{\omega}^{1}(G), \pi_{\ell}\left(r_{i} f\right)=0, i=1, \cdots, d\right\} \\
& =\left\{f \in L_{\omega}^{1}(G), \int_{N} \chi_{p}(n) q(s) P_{i}(s n) f(s n) d n=0, i=1, \cdots, d,\right. \\
& \text { for almost all } s \in S\} \\
& =\left\{f \in L_{\omega}^{1}(G), \int_{N} \chi_{p}(n) p_{i}(n) f(s n) d n=0, i=1, \cdots, d,\right. \\
& \text { for almost all } s \in S\} \\
& =\left\{f \in L_{\omega}^{1}(G), \int_{N} \psi(n) f(n s) d n=0,\right. \\
& \text { for almost all } \left.s \in S, \text { for all } \psi \in j\left(\chi_{p}\right)^{\perp}\right\} \\
& =\left\{f \in L_{\omega}^{1}(G), \int_{N} \psi(n) f * \beta(n) d n=0 \text {, for all } \beta \in C_{c}(G)\right. \text {, } \\
& \text { for all } \left.\psi \in j\left(\chi_{p}\right)^{\perp}\right\} \\
& =\left\{f \in L_{\omega}^{1}(G), f \in e_{G}\left(j\left(\chi_{p}\right)\right)\right\} \\
& =j(\Omega)\left(=j\left(\pi_{\ell}\right)\right) \text { by formula (3.2). }
\end{aligned}
$$

Let $\mathcal{S}\left(G / N, \chi_{\ell}\right)$ be the convolution algebra

$$
\begin{aligned}
\mathcal{S}\left(G / N, \chi_{\ell}\right)= & \{f: G \rightarrow \mathbb{C}, f \text { smooth }, f \circ \exp \in \mathcal{S}(\mathfrak{s}), \\
& \left.f(g n)=\overline{\chi_{p}(n)} f(g), g \in G, n \in N\right\} .
\end{aligned}
$$

The mapping

$$
\Psi: \mathcal{S}(G) \rightarrow \mathcal{S}\left(G / N, \chi_{\ell}\right) ; \Psi(f)(g):=\int_{N} f(g n) \chi_{p}(n) d n, g \in G, f \in \mathcal{S}(G)
$$

is a surjective homomorphism. By Remark 3.13. we can map $\mathcal{S}\left(G / N, \chi_{\ell}\right)$ bijectively onto $\mathcal{B}\left(\mathcal{H}_{\pi_{\ell}}\right)^{\infty}$ using the mapping

$$
\tilde{\pi}_{\ell}(f):=\int_{G / N} f(g) \pi_{\ell}(g) d \dot{g}
$$

As in Remark 3.7, we choose for every $j=1, \cdots, d$ a function $\delta_{j} \in \mathcal{S}(N)$, such that $\int_{N} p_{i}(n) \delta_{j}(n) \chi_{p}(n) d n=\delta_{i j}, i=1, \cdots, d$. If we take any $A_{j} \in \mathcal{B}\left(\mathcal{H}_{\pi_{\ell}}\right)^{\infty}$, then we can find by $\left[\mathrm{Ho}\right.$ an $f_{j} \in \mathcal{S}\left(G / N, \chi_{l}\right)$, such that $\tilde{\pi}_{\ell}\left(f_{j}\right)=A_{j}, j=1, \cdots, d$. Let $\alpha_{i}$ be the Schwartz function on $G$ defined by

$$
\alpha_{i}(s n):=q(s)^{-1} f_{i}(s) \delta_{i}(n)=\mu(s) f_{i}(s) \delta_{i}(n), s \in S, n \in N(i=1, \cdots, d) .
$$


It follows for the function $k:=\sum_{i=1}^{d} \alpha_{i} \in \mathcal{S}(G)$ that

$$
\begin{aligned}
\pi_{\ell}\left(r_{j} k\right) & =\pi_{\ell}\left(\sum_{i=1}^{d} r_{j} \alpha_{i}\right) \\
& =\sum_{i=1}^{d} \int_{S} \int_{N} \pi_{\ell}(s n) r_{j}(s n) \alpha_{i}(s n) d n d s \\
& =\sum_{i=1}^{d} \int_{S} \int_{N} \pi_{\ell}(s) \chi_{p}(n) q(s) p_{j}(n) q(s)^{-1} f_{i}(s) \delta_{i}(n) d n d s \\
& =\sum_{i=1}^{d} \int_{S} \pi_{\ell}(s) f_{i}(s) \int_{N} p_{j}(n) \delta_{i}(n) \chi_{p}(n) d n \\
& =\int_{S} \pi_{\ell}(s) f_{j}(s) d s \\
& =\tilde{\pi}_{\ell}\left(f_{j}\right) \\
& =A_{j} .
\end{aligned}
$$

Hence $\Phi$ maps $\mathcal{S}(G)$ onto the space $\left(B\left(\mathcal{H}_{\ell}\right)^{\infty}\right)^{d}$.

Now take $f \in I^{\infty}$. The function $f$ may for instance be of the form $f=\alpha * k * \beta$ for $k \in I$ and $\alpha, \beta \in C_{c}^{\infty}(G)$. Since the functions $u * r_{j}$ and $r_{j} * u(u \in \mathcal{U}(\mathfrak{g}))$ are bounded by $\omega$ by Lemma 3.12, the functions $r_{j} f$ are in $L^{1}(G)^{\infty}$, and therefore $\pi_{\ell}\left(r_{j} f\right) \in \mathcal{B}\left(\mathcal{H}_{\ell}\right)^{\infty}, j=1, \cdots, d$. Hence $\Phi(f) \in\left(\mathcal{B}\left(\mathcal{H}_{\ell}\right)^{\infty}\right)^{d}$ and so there exists $f^{\prime} \in \mathcal{S}(G)$ such that $\Phi\left(f^{\prime}\right)=\Phi(f)$. Hence $f^{\prime}-f \in \operatorname{ker}(\Phi)=j\left(\pi_{\ell}\right)$. Since $I \supset j\left(\pi_{\ell}\right)$, it follows that $f^{\prime} \in I$, and so $f^{\prime} \in I^{S}$. Because $j\left(\pi_{\ell}\right)$ is the minimal ideal with hull $\left\{\pi_{\ell}\right\}$, we also have that $j\left(\pi_{\ell}\right) \cap \mathcal{S}(G)$ is dense in $j\left(\pi_{\ell}\right)$, and hence for any $\varepsilon>0$ there exists $f_{\varepsilon} \in j\left(\pi_{\ell}\right) \cap \mathcal{S}(G) \subset I^{S}$ such that $\left\|f-f^{\prime}-f_{\varepsilon}\right\|_{1, \omega}<\varepsilon$. But $f^{\prime}+f_{\varepsilon} \in I^{S}$. This shows that $f \in \overline{I^{S}}$ and finally $I=\overline{I^{S}}$.

Remark 3.14. Let $J \subset L_{\omega_{\mid N}}^{1}(N)$ be a two-sided $G$-invariant closed ideal such that $J=\overline{J^{S}}$. Then it is easy to see that the two-sided closed ideal $I=e_{G}(J)$ has the same property, i.e. $I=\overline{I^{S}}$. Indeed, an element $f \in I$ can be approximated by functions of the form $a * \beta, a \in J, \beta \in C_{c}^{\infty}(G)$, and we can approximate the function $a$ by Schwartz functions $b \in J^{S}$; hence $f$ is approximated by the $b * \beta$ 's in $I^{S}$.

\section{The $G \times G$-Module $\mathcal{S}\left(G / N, \chi_{\ell}\right)$}

The Hilbert space $L^{2}\left(G / N, \chi_{\ell}\right)$ is a $G \times G$-module for the presentation $\lambda$ of $G \times G$, where $\lambda$ is defined by

$$
\lambda\left(g, g^{\prime}\right) \xi(x):=\xi\left(g^{-1} x g^{\prime}\right), x, g, g^{\prime} \in G, \xi \in L^{2}\left(G / N, \chi_{\ell}\right) .
$$

Let $\mathfrak{h}$ be a polarization at $\ell$ and let $H:=\exp (\mathfrak{h})$. Then the mapping

$$
\begin{aligned}
\mathcal{S}\left(G / N, \chi_{\ell}\right) & \rightarrow \mathcal{S}\left(G^{2} / H^{2}, \chi_{\ell,-\ell}\right) \\
f & \mapsto U(f):=f_{\ell} \text { given by } f_{\ell}\left(g, g^{\prime}\right):=\int_{H / N} f\left(g h\left(g^{\prime}\right)^{-1}\right) \chi_{\ell}(h) d \dot{h}, g^{\prime}, g \in G,
\end{aligned}
$$


respects the $L^{2}$ norms since by [Lu3], for $f \in \mathcal{S}\left(G / N, \chi_{\ell}\right)$,

$$
\int_{G / H \times G / H}\left|f_{\ell}(u, v)\right|^{2} d \dot{u} d \dot{v}=\left\|\pi_{\ell}(f)\right\|_{\mathrm{HS}}^{2}=\int_{\mathfrak{n}^{\perp}}|\widehat{f}(\ell+p)|^{2} d p=\int_{G / N}|f(x)|^{2} d \dot{x} .
$$

Hence the mapping $U$ extends to an isometric intertwining operator for the representations $\lambda$ and $\pi_{\ell} \times \pi_{-\ell}$ of $G \times G$, and therefore the representation $\lambda$ is itself irreducible (see [Lu3]).

Proposition 4.1. Let $\Omega$ be a flat $G$-orbit in $\mathfrak{g}^{*}$. Let $\left(\pi_{\ell}, \mathcal{H}_{\ell}\right)$ be the irreducible unitary representation of $G$ associated with $\Omega$. Then the Fréchet space $\mathcal{S}\left(G / N, \chi_{p}\right)$ is a simple $\mathcal{S}(G \times G)$-module under left and right convolution.

Proof. Since the orbit $\Omega$ is flat, we have that

$$
\operatorname{ker}\left(\pi_{\ell}\right) \cap \mathcal{S}(G)=\left\{f \in \mathcal{S}(G), \int_{N} f(x n) \chi_{\ell}(n) d n=0, \forall x \in G\right\} .
$$

On the other hand, denoting by $H S_{\ell}$ the Hilbert space of the Hilbert-Schmidt operators on $\mathcal{H}_{\ell}$, it follows from $[\mathrm{Ho}$, that the map

$$
\begin{gathered}
\mathcal{S}(G) \quad \rightarrow\left(H S_{\ell}\right)^{\infty}, \\
f \\
\mapsto \pi_{l}(f)
\end{gathered}
$$

is surjective and the $\mathcal{S}(G \times G)$-module $H S_{\ell}^{\infty}$ is simple. Hence the algebra $\mathcal{S}(G \times G)$ acting by left and right convolution on $\mathcal{S}(G) /\left(\operatorname{ker}(\pi)_{l} \cap \mathcal{S}(G)\right)$ gives us a simple module which is equivalent to the module $\mathcal{S}\left(G / N, \chi_{\ell}\right)$.

Remark 4.2. Let $n \in \mathbb{N}^{*}$ and $f_{1}, \cdots, f_{n}, g_{1}, \cdots, g_{n} \in \mathcal{S}(G)$ such that the functions $\Psi f_{1}, \cdots, \Psi f_{n} \in \mathcal{S}\left(G / N, \chi_{\ell}\right)$ are linearly independent. Then there exists $F \in$ $\mathcal{S}(G \times G)$ such that

$\int_{N} g_{j}(x n) \chi_{\ell}(n) d n=\int_{G \times G} \int_{N} F(u, v) f_{j}\left(u^{-1} x v n\right) \chi_{\ell}(n) d n d u d v, x \in G, j=1, \cdots, n$.

This follows immediately from the simplicity of the $\mathcal{S}(G \times G)$-module $\mathcal{S}\left(G / N, \chi_{\ell}\right)$ and hence from the $n$-transitivity of the $\mathcal{S}(G \times G)$-module $\mathcal{S}\left(G / N, \chi_{\ell}\right)$.

\section{Definition 4.3.}

(1) Let us write for an $F \in \mathcal{S}(G \times G)$ and a measurable bounded function $h$ on $G$ :

$$
F \cdot h(g):=\lambda(F)(h)(g)=\int_{G \times G} F(u, v) h\left(u^{-1} g v\right) d u d v .
$$

(2) We do the same for $A \in \mathcal{U}(\mathfrak{g} \times \mathfrak{g})$ and a smooth function $\varphi$ on $G$. We let $A \cdot \varphi$ denote the differential of the natural action of $G \times G$ on the functions on $G$.

(3) Using the cross section introduced in section 2 we introduce the coordinates $\tau(g)$ and $\eta(g)$ on $G$ :

$$
g=\tau(g) \eta(g),
$$

where $\tau(g)$ denotes the $S$-component of $g$ and $\eta(g)$ its $N$-component.

(4) For a polynomial function $p: N \rightarrow \mathbb{C}, p \in \mathcal{P}_{\omega_{\mid N}}$, let

$$
\Psi_{p}(f)(x):=\int_{N} p(n) f(x n) \chi_{\ell}(n) d n, f \in \mathcal{S}(G), x \in G .
$$


Again take a Jordan-Hölder basis $\left(p_{j}\right)_{j=0}^{d}$ of $\mathcal{P}_{\omega_{\mid N}}(N)$ for the $G \times N$-action. Also let

$$
W_{0}=\mathbb{C} \subset W_{1} \subset \cdots \subset W_{d}=\mathcal{P}_{\omega_{\mid N}}(N)
$$

be the corresponding composition series of the $G \times N$-module $\mathcal{P}_{\omega_{\mid N}}(N)$. For one of the basis polynomials $p_{j}, j=0, \cdots, d$, we simply write

$$
\Psi_{j} \text { instead of } \Psi_{p_{j}} \text {. }
$$

If we express the left translation of $p$ in the basis $\left(p_{j}\right)_{j}$, i.e. if we put

$$
l(m) p_{j}(n)=\sum_{i=0}^{d} l_{i, j}(m) p_{i}(n), n, m \in N, j=1, \cdots, d
$$

and similarly, for $g \in G$ let

$$
\sigma(g) p_{j}=\sum_{i=0}^{j} \sigma_{i, j}(g) p_{i}, j=1, \cdots, d,
$$

then we obtain the relations

$$
\begin{aligned}
\Psi_{j}(f)(x m) & =\int_{N} p_{j}(n) f(x m n) \chi_{\ell}(n) d n \\
& =\int_{N} p_{j}\left(m^{-1} n\right) f(x n) \chi_{\ell}\left(m^{-1} n\right) d n \\
& =\sum_{i=0}^{j} l_{i, j}(m) \chi_{\ell}\left(m^{-1}\right) \Psi_{i}(f)(x) .
\end{aligned}
$$

In the following proposition, we use the notation $I_{V}$ introduced in Theorem 3.2 and $I^{S}$ introduced in Definition 3.9 .

Proposition 4.4. Let $j \in\{1, \cdots, d\}$ and $f \in I_{W_{j-1}}^{S}$. Then for any $F \in \mathcal{S}(G \times G)$, we have that

$$
F \cdot \Psi_{j}(f)=\Psi_{j}(F \cdot f), \Psi_{i}(F \cdot f)=0, i=1, \cdots, j-1
$$

In particular, the mapping $\Psi_{j}: I_{W_{j-1}}^{S} \rightarrow \mathcal{S}\left(G / N, \chi_{\ell}\right)$ is an $\mathcal{S}(G \times G)$-equivariant surjection with kernel $I_{W_{j}}^{S}$ and the $\mathcal{S}(G \times G)$-module $I_{W_{j-1}}^{S} / I_{W_{j}}^{S}$ is equivalent to the module $\mathcal{S}\left(G / N, \chi_{\ell}\right)$ and is therefore simple. 
Proof. Now take $f \in \operatorname{ker}\left(\pi_{\ell}\right) \cap \mathcal{S}(G)$. Then:

$$
\begin{aligned}
\Psi_{j}(F \cdot f)(x) & =\int_{N} p_{j}(n) F \cdot f(x n) \chi_{\ell}(n) d n \\
& =\int_{N} p_{j}(n) \int_{G \times G} F(u, v) f\left(u^{-1} x n v\right) d u d v \chi_{\ell}(n) d n \\
& =\int_{G \times G}\left(\int_{N} p_{j}\left(v n v^{-1}\right) f\left(u^{-1} x v n\right) \chi_{\ell}(n) d n\right) F(u, v) d u d v \\
& =\sum_{i=1}^{j} \int_{G \times G}\left(\int_{N} p_{i}(n) f\left(u^{-1} x v n\right) \chi_{\ell}(n) d n\right) \sigma_{i, j}\left(v^{-1}\right) F(u, v) d u d v \\
& =\sum_{i=1}^{j} F_{i, j} \cdot \Psi_{i}(f)(x), x \in G,
\end{aligned}
$$

where $F_{i, j}(u, v)=\sigma_{i, j}\left(v^{-1}\right) F(u, v), u, v \in G, i \leq j$. Now suppose that $f \in I_{W_{j-1}}^{S} \backslash I_{W_{j}}^{S}$. Then we have that

$$
0=\int_{N} p_{i}(n) f\left(u^{-1} x v n\right) \chi_{\ell}(n) d n, i=1, \cdots, j-1, x, u, v \in G,
$$

and therefore, for $F \in \mathcal{S}(G \times G)$,

$$
\Psi_{j}(f) \in \mathcal{S}\left(G / N, \chi_{\ell}\right) \text { and } F \cdot\left(\Psi_{j} f\right)=\Psi_{j}(F \cdot f),
$$

as $\sigma_{j, j} \equiv 1$.

\section{The Structure of PRIMARY IDEALS With A Flat HUlL}

Let $I \in \mathcal{I}^{\pi_{\ell}}$ such that $W(I) \subsetneq V(I)$. Let us recall that the notation $W(I)$ and $V(I)$ were introduced in the relation of Remark 3.3 and that the strict inclusion means that $I$ is not $L^{\infty}(G / N)$-invariant, by Proposition 2.3. Choose a composition series $W_{0}=W(I) \subset \cdots \subset W_{r}=V(I)$ for the action of $G \times N$ on $\mathcal{P}_{\omega_{\mid N}}(N)$, going through $W(I)$ and $V(I)$. Since the action of $G \times N$ is unipotent on $\mathcal{P}_{\omega_{\mid N}}(N)$ it follows that $\operatorname{dim}\left(W_{j} / W_{j-1}\right)=1$ for every $j$. Furthermore,

$$
I_{V(I)}=I_{W_{r}} \subsetneq I_{W_{r-1}} \subsetneq \cdots \subsetneq I_{W_{0}}=I_{W(I)} .
$$

Choose a basis $\mathcal{B}:=\left\{p_{1}, \cdots, p_{r}\right\}$ of $V(I)$ modulo $W(I)$, which is adapted to the sequence $\left(W_{j}\right)_{j=1}^{r}$, i.e., $W_{j}=\sum_{i=1}^{j} \mathbb{R} p_{i}+W(I), j=1, \cdots, r$. Also let $\left(q_{j}\right)_{j=1}^{s}$ be a Jordan-Hölder basis of the $G \times N$-module $W(I)$ and similarly a Jordan-Hölder basis $\left(r_{j}\right)_{j=1}^{t}$ of the $G \times N$-module $\mathcal{P}_{\omega_{\mid N}}(N) / V(I)$.

Definition 5.1. Define for a function $h \in \mathcal{S}(S)$ and a function $\delta \in \mathcal{S}(N)$ the Schwartz function $f_{h, \delta}=\mathfrak{h} \otimes \delta$ on $G$ by

$$
f_{h, \delta}(x)=h \otimes \delta(x):=h(\tau(x)) \delta(\eta(x)), x \in G .
$$

Proposition 5.2. Let $W \subset V$ be two $G \times N$-invariant subspaces in $\mathcal{P}_{\omega_{\mid N}}(N)$. Choose for every $j=1, \cdots, r$ a function $\delta_{j} \in \mathcal{S}(N)$ such that

$$
\begin{aligned}
\int_{N} p_{i}(n) \delta_{j}(n) \chi_{\ell}(n) d n & =\delta_{i j}, i, j=1, \cdots, r \\
\int_{N} p(n) \delta_{j}(n) \chi_{\ell}(n) d n & =0, p \in W,
\end{aligned}
$$


where $\left\{p_{1}, \cdots, p_{r}\right\}$ is a Jordan-Hölder basis of $V \bmod W$ for the action of $G \times N$. Then for every $j=1, \cdots, r$ we have that

$$
I_{W}^{\infty}=\sum_{j=1}^{r} \mathcal{S}(S) \otimes \delta_{j}+I_{V}^{\infty} .
$$

Let $f \in I_{W}^{\infty}$ and as before let

$$
\Psi_{j}(f)(x)=\int_{N} f(x n) p_{j}(n) \chi_{\ell}(n) d n, x \in G, j=1, \cdots, r .
$$

Define for $j=1, \cdots, r$ the Schwartz function $f_{j}(x):=\Psi_{j}(f)(\tau(x)) \delta_{j}(\eta(x)), x \in G$. Then

$$
f=\sum_{j=1}^{r} f_{j} \bmod I_{V} .
$$

Proof. The ideal $I_{V}:=e_{G}\left(J_{V}\right)$ (see Theorem 3.2) is determined by the condition

$$
I_{V}=\left\{f \in L_{\omega}^{1}(G), \int_{S} \int_{N} \varphi(s) p(n) \chi_{\ell}(n) f(s n) d n d s=0, \varphi \in C_{c}(G / N), p \in V\right\} .
$$

Hence

$$
I_{V}^{S}=I_{V} \cap \mathcal{S}(G)=\left\{f \in \mathcal{S}(G) \cap I_{W} ; \Psi_{j}(f)=0, j=1, \cdots, r\right\} .
$$

Moreover, by Theorem 3.11, $I_{W}^{\infty}$ is contained in $\mathcal{S}(G)$ modulo $j(\Omega)$. Hence we may use Schwartz functions in the following arguments. By the definition of the functions $f_{j}$, we have for $k=1, \cdots, r$ that

$$
\begin{aligned}
& \int_{N}\left(f(x n)-\sum_{j=1}^{r} f_{j}(x n)\right) p_{k}(n) \chi_{\ell}(n) d n \\
= & \int_{N} f(x n) p_{k}(n) \chi_{l}(n) d n-\sum_{j=1}^{r} \Psi_{j}(f)(\tau(x)) \int_{N} \delta_{j}(\eta(x) n) p_{k}(n) \chi_{\ell}(n) d n \\
= & \Psi_{k}(f)(x)-\sum_{j=1}^{r} \Psi_{j}(f)(\tau(x)) \chi_{\ell}\left(\eta(x)^{-1}\right) \int_{N} \delta_{j}(n) p_{k}\left(\eta(x)^{-1} n\right) \chi_{\ell}(n) d n \\
= & \Psi_{k}(f)(x)-\sum_{j=1}^{r} \Psi_{j}(f)(\tau(x)) \chi_{\ell}\left(\eta(x)^{-1}\right) \int_{N} \delta_{j}(n)\left(\sum_{i=1}^{r} l_{i, k}(\eta(x)) p_{i}(n)\right) \chi_{\ell}(n) d n \\
= & \Psi_{k}(f)(x)-\sum_{j=1}^{r} l_{j, k}(\eta(x)) \Psi_{j}(f)(\tau(x)) \chi_{l}\left(\eta(x)^{-1}\right) \\
= & \Psi_{k}(f)(x)-\int_{N} f\left(\tau(x) \eta(x) \eta(x)^{-1} n\right) p_{k}\left(\eta(x)^{-1} n\right) \chi_{l}\left(\eta(x)^{-1} n\right) d n \\
= & \Psi_{k}(f)(x)-\Psi_{k}(f)(x) \\
= & 0 .
\end{aligned}
$$

Hence $\Psi_{k}\left(f-\sum_{j} f_{j}\right)=0$ and therefore $f=\sum_{j} f_{j}$ modulo $I_{V}$, as $f-\sum_{j} f_{j} \in$ $\mathcal{S}(G) \cap I_{W}$.

Remark 5.3. It is easy to see that there exists for $j=1, \cdots, r$ an element $f \in$ $I \cap \mathcal{S}(G)$ such that the function $\Psi_{j} f$ is linearly independent from the functions 
$\Psi_{i} f, i=j-1, \cdots, 1$. Indeed consider for $p \in V(I)$ the linear functional $\bar{p} \in(I \cap$ $\mathcal{S}(G))^{\prime}$ defined by

$$
\langle\bar{p}, f\rangle:=\int_{N} f(n) p(n) \chi_{\ell}(n) d n .
$$

Then the kernel of the functional $\overline{p_{j}}$ does not contain the intersection of the kernels of the functionals $\overline{p_{i}}, j=1, \cdots, j-1$, since otherwise, $\overline{p_{j}}=\sum_{i=j-1}^{1} \alpha_{i} \overline{p_{i}}$ for some coefficients $\alpha_{i} \in \mathbb{C}$. But this would imply that $p_{j}-\sum_{i=j-1}^{1} \alpha_{j} p_{i} \in W(I)$, i.e. $p_{j} \in W_{j-1}$. We now take a function $f \in I \cap \mathcal{S}(G)$ such that $\left\langle\overline{p_{i}}, f\right\rangle=0$ for all $i=1, \cdots, j-1$ and $\left\langle\overline{p_{j}}, f\right\rangle=1$. Then

$$
\Psi_{j} f(e)=1, \Psi_{i} f(e)=0, i=1, \cdots, j-1,
$$

and so $\Psi_{j} f$ is not contained in $\operatorname{span}\left\{\Psi_{1} f, \cdots, \Psi_{j-1} f\right\}$.

Definition 5.4. (1) For a function $f \in \mathcal{S}(G)$, let

$$
\langle f\rangle:=\{F \cdot f, F \in \mathcal{S}(G \times G)\} .
$$

(2) Let $I$ be a closed two-sided ideal in $\mathcal{I}^{\pi_{\ell}}$ such that $V(I) \neq W(I)$, and let

$$
\mathcal{C}:=\left\{W(I)=W_{0} \subset W_{1} \subset \cdots \subset W_{r}=V(I)\right\}
$$

be a composition series of $V(I) / W(I)$ for the $G \times N$-action. We define the index set

$$
\begin{aligned}
\iota_{\mathcal{C}}^{I}=\iota^{I} & :=\left\{1 \leq i \leq r ; I \cap I_{W_{i}} \subsetneq I \cap I_{W_{i-1}}\right\} \\
& =\left\{j_{1}<\cdots<j_{m}\right\} .
\end{aligned}
$$

(3) We denote by $I^{\infty}$ the space of the smooth vectors (for the action of $G \times G$ ) of $I \subset L^{1}(G, \omega)$.

Theorem 5.5. Let $\Omega=\operatorname{Ad}^{*}(G) \ell=\ell+\mathfrak{g}(\ell)^{\perp}$ be a flat orbit in the dual space $\mathfrak{g}^{*}$ of the Lie algebra of the simply connected connected nilpotent Lie group $G$. Let $\omega$ be a continuous symmetric polynomial weight on $G$. Then for every two-sided closed ideal I in the Banach algebra $L_{\omega}^{1}(G)$ whose hull is the set $\{\Omega\}$, we have that $I$ is $L^{\infty}(G / N)$-invariant if and only if $V(I)=W(I)$. If $V(I) \supsetneq W(I)$, then

$$
I_{V(I)} \subsetneq I \subsetneq I_{W(I)} \text {. }
$$

Furthermore, for every composition series

$$
\mathcal{C}=\left\{W(I)=W_{0} \subset W_{1} \subset \cdots \subset W_{r}=V(I)\right\}
$$

of the $G \times N$-module $V(I) / W(I)$, the index set $\iota_{\mathcal{C}}^{I}$ is contained in $\{1, \cdots, r-1\}$ and contains the number 1 .

If we choose for any $i \in \iota^{I}$ an element $f_{i} \in I^{S} \cap I_{W_{i-1}} \backslash I_{W_{i}}$, then we have that

$$
I^{\infty}=\sum_{i \in \iota_{I}}\left\langle f_{i}\right\rangle+I_{V(I)}^{\infty} .
$$

In particular, if for two ideals $I_{1}, I_{2} \in \mathcal{I}^{\pi_{\ell}}$ such that $V\left(I_{1}\right)=V\left(I_{2}\right)$ and $W\left(I_{1}\right)=$ $W\left(I_{2}\right)$ the index sets $\iota^{I_{1}}=\iota^{I_{2}}$ coincide and if $I_{1} \subset I_{2}$, then $I_{1}=I_{2}$.

Proof. We have that

$$
I_{V(I)}=I_{W_{r}} \subset I \cap I_{W_{r-1}} \subset \cdots \subset I \cap I_{W_{1}} \subset I \cap I_{W_{0}}=I \subset I_{W(I)} .
$$


Also choose for every $i \in \iota^{I}$ an element $f_{i} \in I^{S} \cap I_{W_{i-1}} \backslash I_{W_{i}}$. Hence by Proposition 4.4, we have that

$$
\left\{F \cdot f_{i}, F \in \mathcal{S}(G \times G)\right\}+\left(I_{W_{i}}\right)^{S}=\left(I_{W_{i-1}}\right)^{S}, i \in \iota^{I} .
$$

Hence for every $g \in I^{S}$, we have an $F_{j_{1}} \in \mathcal{S}(G \times G)$ such that $g-F_{j_{1}} \cdot f_{j_{1}} \in I \cap I_{W_{j_{1}}}$. Continuing in this way we find $F_{i} \in \mathcal{S}(G \times G), i \in \iota^{I}$, such that

$$
g-\sum_{i \in \iota^{I}} F_{i} \cdot f_{i} \in\left(I_{W_{r}}\right)^{S}=\left(I_{V(I)}\right)^{S} .
$$

Therefore

$$
I^{S}=I \cap \mathcal{S}(G)=\sum_{i \in \iota^{I}}\left\langle f_{i}\right\rangle+\left(I_{V(I)}\right)^{S} .
$$

Now recall that every $C^{\infty}$-vector $f \in I$ is contained in $I^{S}$ modulo $j\left(\pi_{\ell}\right)^{\infty}$. Hence

$$
I^{\infty}=\sum_{i \in \iota^{I}}\left\langle f_{i}\right\rangle+I_{V(I)}^{\infty} .
$$

Let us show that the index set $\iota_{\mathcal{C}}^{I}$ is contained in $\{1, \cdots, r-1\}$.

According to Remark 3.7, we have for every $\varphi \in I^{\perp} \cap C_{\omega}^{\infty}(G)$ the expression

$$
\varphi(s n)=\sum_{j=1}^{r} \varphi_{j}(s) p_{j}(n) \chi_{\ell}(n), s \in S, n \in N \text {, modulo } W(I) \text {. }
$$

Now if $j_{m}=r$, then for $g \in I_{W_{r-1}}$ we have our $F_{g} \in \mathcal{S}(G \times G)$ and our $f_{m}=f_{j_{m}} \in$ $I \cap I_{W_{r-1}}^{S} \backslash I_{W_{r}}^{S}$, such that $g=F_{g} \cdot f_{j_{m}} \bmod I_{W_{r}}=I_{V(I)}$. Hence, by Proposition 4.4. $\Psi_{r}(g)=F_{g} \cdot \Psi_{r}\left(f_{m}\right), \Psi_{j}(g)=0$ for $j<r$, and therefore for $\varphi \in I^{\perp} \cap C_{\omega}^{\infty}(G)$,

$$
\begin{aligned}
\langle\varphi, g\rangle & =\int_{S} \int_{N} \varphi(s n) g(s n) d n d s \\
& =\int_{S} \sum_{j=1}^{r} \varphi_{j}(s) \Psi_{j}(g)(s) d s \\
& =\int_{S} \varphi_{r}(s) \Psi_{r}(g)(s) d s \\
& =\int_{S} \varphi_{r}(s) F_{g} \cdot \Psi_{r}\left(f_{m}\right)(s) d s \\
& =\int_{S} \varphi_{r}(s) \Psi_{r}\left(F_{g} \cdot f_{m}\right)(s) d s \text { (by Proposition 4.4) } \\
& =\int_{S} \sum_{j=1}^{r} \varphi_{j}(s) \Psi_{j}\left(F_{g} \cdot f_{m}\right)(s) d s \\
& =\left\langle\varphi, F \cdot f_{m}\right\rangle \\
& =0 .
\end{aligned}
$$

Hence $I_{W_{r-1}} \subset I$ and $I_{V(I)}=I_{W_{r}} \subsetneq I_{W_{r-1}} \subset I$ contradicts the fact that $I_{V(I)}$ is the largest $L^{\infty}(G / N)$-invariant ideal with hull $\Omega$ contained in $I$. This contradiction tells us that $j_{m}<r$.

Now if $j_{1} \neq 1$, then $I=I \cap I_{W(I)}=I \cap I_{W_{1}}$, and therefore $I \subset I_{W_{1}}$. But then $W_{1} \subset W(I)$, a contradiction. 


\section{The Case of a Central stabilizer}

Lemma 6.1. Let $\Omega$ be a flat $G$-orbit. Let $V$ be $a G \times N$ invariant subspace of $\mathcal{P}_{\omega_{\mid N}}(N)$. Let $I$ be a closed two-sided ideal in $L_{\omega}^{1}(G)$ such that the hull of $I$ is the set $\Omega$ and such that $V(I) \subset V$. If $I_{V} \subsetneq I$ then there exists a function $f \in \mathcal{S}(G) \cap I$ such that $f \notin I_{V}$, but such that $(u+i \ell(u)) * f \in I_{V}$ for every $u \in \mathfrak{n}$. In particular, for any $p \in V$, we have that

$$
\int_{N} p(m n) f(g n v) \chi_{\ell}(n) d n=\int_{N} p(n) f(g n v) \chi_{\ell}(n) d n, \text { for every } g, v \in G, m \in N .
$$

Proof. Take a Jordan-Hölder sequence

$$
W(I)=V_{0} \subset V_{1} \subset \cdots \subset V_{i}=V(I) \subset \cdots \subset V_{r}=V
$$

for the action of $G \times N$ on $\mathcal{P}_{\omega_{\mid N}}(N)$. As $I_{V} \subsetneq I, W(I) \subsetneq V$, and hence $W(I) \subsetneq V_{1}$. Let $f \in \mathcal{S}(G) \cap I$ and $p \in V_{1} \backslash W(I)$. For $u \in \mathfrak{n}$ we see that

$$
\begin{aligned}
\int_{N} p(n)(u+i \ell(u)) * f(g n v) \chi_{\ell}(n) d n & =-\int_{N}\left(\operatorname{Ad}\left(g^{-1}\right)(u) * p\right)(n) f(g n v) \chi_{\ell}(n) d n \\
& =0,
\end{aligned}
$$

since $\operatorname{Ad}\left(g^{-1}\right)(u) * p \in W(I)$, because $u$ acts as a nilpotent endomorphism on $\mathcal{P}_{\omega_{\mid N}}(N)$. Continuing in this way, we see that we can find for $f \in I^{S} \backslash I_{V}$ an element $A \in \mathcal{U}(\mathfrak{n})$ such that $A * f \notin I_{V}$, but such that $(u+i \ell(u)) * A * f \in I_{V}$ for every $u \in \mathfrak{n}$. Hence replacing $f$ with $A * f$, we can in this way produce an element $f \in \mathcal{S}(G) \cap I$, which is not contained in $I_{V}$, but such that $(u+i \ell(u)) * f \in I_{V}$ for every $u \in \mathfrak{n}$. Hence for any $p \in V$, for any $g, v \in G, u \in \mathfrak{n}$,

$$
\begin{array}{rl}
\int_{N} u & * p(n) f(g n v) \chi_{\ell}(n) d n \\
& =\left.\frac{d}{d t} \int_{N} p(\exp (-t u) n) f(g n v) \chi_{l}(n) d n\right|_{t=0} \\
& =\int_{N} p(n)(-\operatorname{Ad}(g)(u)) * f(g n v) \chi_{l}(n) d n-i l(u) \int_{N} p(n) f(g n v) \chi_{l}(n) d n \\
& =-\int_{N} p(n)(\operatorname{Ad}(g)(u)+i l(\operatorname{Ad}(g)(u))) * f(g n v) \chi_{l}(n) d n \\
& =0
\end{array}
$$

as $l(\operatorname{Ad}(g)(u))=l(u)$. Now for $m \in N, p \in V(I)$, we have that $p(m n)=p(n)+$ $q_{m}(n)$ for some polynomial $q_{m}$, which is obtained from $p$ via some derivatives by elements of $\mathfrak{n}$. It then follows that

$$
\begin{aligned}
\int_{N} p(m n) f(g n v) \chi_{\ell}(n) d n & =\int_{N}\left(p(n)+q_{m}(n)\right) f(g n v) \chi_{\ell}(n) d n \\
& =\int_{N} p(n) f(g n v) \chi_{\ell}(n) d n, g, v \in G .
\end{aligned}
$$

Proposition 6.2. Let $\Omega=\operatorname{Ad}^{*}(G) \ell=\ell+\mathfrak{g}(\ell)^{\perp}$ be a flat orbit in the dual space $\mathfrak{g}^{*}$ of the Lie algebra of the simply connected connected nilpotent Lie group $G$. Let $\omega$ be a continuous symmetric polynomial weight on $G$. Let $W \subset V$ be two $G \times N$ invariant subspaces in $\mathcal{P}_{\omega_{\mid N}}(N)$. Let $I_{V} \subset I \subset I_{W}$ be a two-sided closed ideal $I$ in 
the Banach algebra $L_{\omega}^{1}(G)$. If the $G$-action on $V / W$ is trivial, then the ideal $I$ is $L^{\infty}(G / N)$-invariant.

Proof. Since $I_{V} \subset I \subset I_{W}$, we have that $W \subset W(I) \subset V(I) \subset V$, and hence the $G$ action on $V(I) / W(I)$ is also trivial. We can therefore assume that $V=V(I), W=$ $W(I)$. If $I=I_{V(I)}$, then $I$ is $L^{\infty}(G / N)$-invariant. So let us assume that $I_{V(I)} \subsetneq I$. Then $W(I) \subsetneq V(I)$ by the relation in Remark 3.3. Also let

$$
\left(I_{0}\right)^{\infty}=\left\{f \in I^{\infty},(u+i l(u)) * f \in I_{V(I)}, \forall u \in \mathfrak{g}\right\}
$$

and let $I_{0}$ be the closure of $\left(I_{0}\right)^{\infty}$ in $L_{\omega}^{1}(G)$. Then $I_{0}$ is a closed two-sided ideal contained in $I$ and containing $I_{V(I)}$ properly by Lemma 6.1 The inclusions $I_{V(I)} \subset$ $I_{0} \subset I$ imply that $V(I) \subset V\left(I_{0}\right) \subset V\left(I_{V(I)}\right)=V(I)$, i.e. $V(I)=V\left(I_{0}\right)$. Moreover, $I_{0} \subset I$ shows that $W(I) \subset W\left(I_{0}\right) \subset V\left(I_{0}\right)=V(I)$.

If $W\left(I_{0}\right)=V\left(I_{0}\right)$, then $I_{0}$ is $L^{\infty}(G / N)$-invariant and $I_{0}=I_{V\left(I_{0}\right)}=I_{V(I)}$, a contradiction.

So let us now assume that $W\left(I_{0}\right) \subsetneq V\left(I_{0}\right)$. Let $\left\{p_{1}, \cdots, p_{r}\right\}$ be a basis of $V\left(I_{0}\right)$ modulo $W\left(I_{0}\right)$. Because of the assumption on the $G$-action and because of the inclusions $W=W(I) \subset W\left(I_{0}\right) \subset V\left(I_{0}\right)=V(I)=V$, the $G$-action on $V\left(I_{0}\right) / W\left(I_{0}\right)$ is also trivial. Moreover, let us notice that for any $p \in V\left(I_{0}\right)=V(I)$,

$$
p\left(\text { munu }^{-1}\right)=p(n)+q_{m, u}(n), n, m \in N, u \in G,
$$

where $q_{m, u}$ is an element of $W\left(I_{0}\right)$. In fact, conjugation is taken care of by the triviality of the $G$-action. Translation by elements of $N$ is taken care of by Lemma 6.1. This means that $\left\{p_{1}, \cdots, p_{r}\right\}$ is a Jordan-Hölder basis for the $G \times N$-action, for any order of the basis vectors.

As before, let us use the notation

$$
\Psi_{j}(f)(s):=\int_{N} f(s n) p_{j}(n) \chi_{\ell}(n) d n, s \in S, f \in I_{W\left(I_{0}\right)} .
$$

Using the fact that the $G$-action on $V\left(I_{0}\right) / W\left(I_{0}\right)$ is trivial, an easy computation shows that

$$
F \cdot \Psi_{j}(f)=\Psi_{j}(F \cdot f), f \in I_{0}, j \in\{1, \cdots, r\}, F \in \mathcal{S}(G \times G) .
$$

Let us now show that $I_{0}=I_{V(I)}$. By Remark 5.3 and by the fact that the order of the basis vectors $p_{1}, \ldots, p_{r}$ does not matter, there exists for every $j \in\{1, \cdots, r\}$ an element $f_{j} \in I_{0}$, for which the function $\Psi_{j}\left(f_{j}\right)$ is linearly independent from the family $\left\{\Psi_{i}\left(f_{j}\right), i \in\{1, \cdots, r, i \neq j\}\right\}$. Finally, the fact that $F \cdot \Psi_{j}(f)=\Psi_{j}(F \cdot f)$ for all $f \in I_{0}, F \in S(G \times G), j \in\{1, \cdots, r\}$, and Remark 4.2 allow us to construct, for every $j \in\{1, \cdots, r\}$, a function $k_{j} \in I_{0}^{S}$ such that $\Psi_{i}\left(k_{j}\right)=0$ for $i \neq j$ and $\Psi_{j}\left(k_{j}\right) \neq 0$. In fact, as the $\Psi_{k}\left(f_{j}\right)$ 's are independent, there exists by Remark 4.2 an $\tilde{F}_{j} \in S(G \times G)$ such that

$$
\begin{aligned}
& \Psi_{k}\left(\tilde{F}_{j} \cdot f_{j}\right)=\tilde{F}_{j} \cdot \Psi_{k}\left(f_{j}\right)=0 \quad \text { if } j \neq k, \\
& \Psi_{j}\left(\tilde{F}_{j} \cdot f_{j}\right)=\tilde{F}_{j} \cdot \Psi_{j}\left(f_{j}\right) \neq 0 .
\end{aligned}
$$

Put $k_{j}=\tilde{F}_{j} \cdot f_{j}$. Now if $h \in I_{W\left(I_{0}\right)}^{\infty}$, then there exists for each $j \in\{1, \cdots, r\}$ an $F_{j} \in S(G \times G)$ such that $h=\sum_{j=1}^{r} F_{j} \cdot k_{j}$ modulo $I_{V\left(I_{0}\right)}$ by Theorem 5.5, But this means that $h \in I_{0}$, since $I_{V\left(I_{0}\right)} \subset I_{0}$ and since the $k_{j}$ 's are all contained in $I_{0}$. So $I_{W\left(I_{0}\right)} \subset I_{0}$ and hence $I_{V\left(I_{0}\right)}=I_{0}=I_{W\left(I_{0}\right)}$, which means that $I_{0}$ is $L^{\infty}(G / N)$ invariant. But then $I_{V(I)}=I_{V\left(I_{0}\right)}=I_{0}$, a contradiction. This contradiction shows that $I_{V(I)}=I$, and hence that $I$ is $L^{\infty}(G / N)$-invariant. 
Theorem 6.3. Let $\Omega=\operatorname{Ad}^{*}(G) \ell=\ell+\mathfrak{g}(\ell)^{\perp}$ be a flat orbit in the dual space $\mathfrak{g}^{*}$ of the Lie algebra of the simply connected connected nilpotent Lie group $G$ such that the stabilizer $\mathfrak{g}(\ell)$ is the centre of $\mathfrak{g}$. Let $\omega$ be a continuous symmetric polynomial weight on $G$. Then for every two-sided closed ideal $I$ in the Banach algebra $L_{\omega}^{1}(G)$ whose hull is the set $\{\Omega\}$, we have that $I$ is $L^{\infty}(G / N)$-invariant, and so $I=e_{G}\left(I_{\mid N}\right)$. In particular, the mapping: $V \rightarrow e_{G}\left(J_{V}\right)$ defines an inclusion reversing bijection between the space $\mathcal{P} \mathcal{I}_{\omega_{\mid N}}(N)^{G}$ of the $G$-invariant and $N$-translation invariant subspaces $V$ of $\mathcal{P}_{\omega_{\mid N}}(N)$ and the space $\mathcal{I}^{\Omega}$ of two-sided closed closed ideals I with hull $\Omega$.

Proof. We have that $I_{V(I)} \subset I \subset I_{W(I)}$ and that $V(I) / W(I)$ is a trivial $G$-module since $\mathfrak{n}$ is central. Hence it suffices to apply Proposition 6.2.

\section{An Algebraic View to some of the PREvious Results}

In this section we consider in particular (the ideal theory in) the topological algebra $\mathcal{S}(G) / \mathcal{S}(G) \cap j\left(\pi_{l}\right)$, which is dense in $L_{\omega}^{1}(G) / j\left(\pi_{l}\right)$. It turns out that $\mathcal{S}(G) / \mathcal{S}(G) \cap j\left(\pi_{l}\right)$ is a Noetherian $\mathcal{S}(G \times G)$-module, for the action of $\mathcal{S}(G \times G)$; see the beginning of section 4 . An important tool will be the existence of an idempotent $\mathfrak{q} \in \mathcal{S}(G) / \mathcal{S}(G) \cap j\left(\pi_{l}\right)$ of finite rank, i.e., $\mathfrak{q} * \mathfrak{q}=\mathfrak{q}$, and $\mathfrak{q} * \mathcal{S}(G) / \mathcal{S}(G) \cap$ $j\left(\pi_{l}\right) * \mathfrak{q}$ is finite-dimensional. This latter space generates the $\mathcal{S}(G \times G)$-module $\mathcal{S}(G) / \mathcal{S}(G) \cap j\left(\pi_{l}\right)$. Actually, $\mathfrak{q} * \mathcal{S}(G) / \mathcal{S}(G) \cap j\left(\pi_{l}\right) * \mathfrak{q}$ is, as a space, isomorphic to $\mathcal{S}(N) / \mathcal{S}(N) \cap j\left(\chi_{p}\right) \cong L_{\omega \mid N}^{1}(N) / j\left(\chi_{p}\right)$. The two-sided closed ideals in $\mathcal{S}(G) / \mathcal{S}(G) \cap j\left(\pi_{l}\right)$ are in one-to-one correspondence to the two-sided ideals in the algebra $\mathfrak{q} * \mathcal{S}(G) / \mathcal{S}(G) \cap j\left(\pi_{l}\right) * \mathfrak{q}$. Finally, the obtained insights will be applied to the ideal theory of $L_{\omega}^{1}(G) / j\left(\pi_{l}\right)$.

We start with an elementary lemma on finite-dimensional nilpotent associative algebras.

Lemma 7.1. Let $\mathcal{R}$ be a d-dimensional associative algebra over the complex number field (or any other field) with unit $\eta$. Let $\mathfrak{r}_{d}=\mathcal{R} \supset \mathfrak{r}_{d-1} \supset \cdots \supset \mathfrak{r}_{1} \supset \mathfrak{r}_{0}=\{0\}$ be a sequence of two-sided ideals with $\operatorname{dim}\left(\mathfrak{r}_{j}\right)=j$ and $\mathfrak{r}_{d-1} \mathfrak{r}_{j} \subset \mathfrak{r}_{j-1}$ and $\mathfrak{r}_{j} \mathfrak{r}_{d-1} \subset \mathfrak{r}_{j-1}$ for $j=1, \cdots, d-1$. Then each proper two-sided ideal in $\mathcal{R}$ is contained in $\mathfrak{r}_{d-1}$, i.e., $\mathfrak{r}_{d-1}$ is the unique maximal ideal. Moreover, $\mathcal{R}=\mathbb{C} \eta \oplus \mathfrak{r}_{d-1}$.

Remark 7.2. If $\mathfrak{m}:=\mathfrak{r}_{d-1}$, then the assumptions tell that $\mathfrak{m}^{d}=0$. In turn, if there is a one-codimensional ideal with this property in some $d$-dimensional algebra with unit, then there always exists a Jordan-Hölder sequence with the above properties: Refine, if necessary, the sequence $\mathfrak{m} \supset \mathfrak{m}^{2} \supset \mathfrak{m}^{3} \supset \cdots$. If $d \geq 2$, then besides the unique maximal ideal $\mathfrak{m}$ there is a canonical "relatively small" ideal $\mathfrak{a}:=\{r \in \mathcal{R} \mid$ $\mathfrak{m} r=r \mathfrak{m}=0\}$ which has the property that $\mathfrak{a} \cap \mathfrak{b} \neq 0$ for any non-zero two-sided $\mathfrak{b}$ in $\mathcal{R}$.

Proof. (Induction on $d$ ) The case $d=1$ being trivial, assume that $d>1$. Let $\mu: \mathcal{R} \rightarrow \mathcal{R} / \mathfrak{r}_{1}$ be the quotient map, and let $J$ be a proper two-sided ideal in $\mathcal{R}$. If $\mu(J) \subsetneq \mathcal{R} / \mathfrak{r}_{1}$ we are done by induction, so assume that $\mu(J)=\mathcal{R} / \mathfrak{r}_{1}$. Then $\mathcal{R}=J \oplus \mathfrak{r}_{1}$; in particular, there exists $h \in J$ and $r \in \mathfrak{r}_{1}$ such that $\eta=h+r$. As $r^{2}=0$, we have $h r=\eta r=r$, and likewise $r h=r$. The equation $h+r=\eta=\eta^{2}$ leads to $h^{2}=h, 2 r=r$; hence $h=\eta \in J$, a contradiction. The equation $\mathcal{R}=\mathbb{C} \eta \oplus \mathfrak{r}_{d-1}$ is obvious. 
Proof of Remark 7.2. If $\mathfrak{b} \neq 0$, then choose $l \geq 0$ such that $\mathfrak{m}^{l} \mathfrak{b} \neq 0$, but $\mathfrak{m}^{l+1} \mathfrak{b}=0$ (if $l=0$, then $\mathfrak{m}^{l}$ is meant to be $\mathcal{R}$ ). Similarly choose $k \geq 0$ such that $\mathfrak{m}^{l} \mathfrak{b m}^{k} \neq 0$, but $\mathfrak{m}^{l} \mathfrak{b m}^{k+1}=0$. Then $\mathfrak{a} \cap \mathfrak{b} \supset \mathfrak{m}^{l} \mathfrak{b m}^{k} \neq 0$.

As in previous sections let $N=G(l), l \in \mathfrak{g}^{*}$, be a member of the flat orbit $\Omega$. As we remarked earlier the minimal ideal $j\left(\chi_{p}\right) \subset L_{\omega \mid N}^{1}(N)$ is the annihilator for the pairing

$$
\langle f, \Phi\rangle=\int_{N} f(x) \chi_{p}(x) \Phi(x) d x,
$$

$f \in L_{\omega \mid N}^{1}(N), \Phi \in \mathcal{P}_{\omega \mid N}(N)$. If we denote by $\mathcal{A}$ the algebra $L_{\omega \mid N}^{1}(N) / j\left(\chi_{p}\right)$, the above pairing induces a non-degenerate duality $\mathcal{A} \times \mathcal{P}_{\omega \mid N}(N) \rightarrow \mathbb{C}$ between the $d$-dimensional spaces $\mathcal{A}$ and $\mathcal{P}_{\omega \mid N}(N)$. The algebra $\mathcal{A}$ has a unique element $\eta$ with the property $\langle\eta, \Phi\rangle=\Phi(e)$ for all $\Phi \in \mathcal{P}_{\omega \mid N}(N)$; this element $\eta$ is the unit in $\mathcal{A}$. Also the other properties of Lemma 7.1 are satisfied. There is a sequence of ideals $\mathfrak{a}_{d}=\mathcal{A} \supset \mathfrak{a}_{d-1} \supset \cdots \supset \mathfrak{a}_{1} \supset \mathfrak{a}_{0}=\{0\}$, where $\mathfrak{a}_{d-1}=\operatorname{ker}\left(\chi_{p}\right) / j\left(\chi_{p}\right)$, such that $\operatorname{dim}\left(\mathfrak{a}_{j}\right)=j$ and $\mathfrak{a}_{d-1} \mathfrak{a}_{j} \subset \mathfrak{a}_{j-1}$ and $\mathfrak{a}_{j} \mathfrak{a}_{d-1} \subset \mathfrak{a}_{j-1}$ for $j=1, \cdots, d-1$. We shall often use $\mathfrak{m}$ for the unique maximal ideal $\mathfrak{a}_{d-1}$.

The right and left translations on $L_{\omega \mid N}^{1}(N)$ with elements of $N$ induce actions $\rho$ and $\lambda$, resp., of $N$ on $\mathcal{A}$, and the two-sided ideals in $\mathcal{A}$ are just the $\lambda$ - $\rho$-invariant subspaces. The dual action $\lambda^{*}$ of $N$ on $\mathcal{P}_{\omega \mid N}(N)$ is given by $\left(\lambda^{*}(n) \Phi\right)(x)=$ $\chi_{p}(n)^{-1} \Phi\left(n^{-1} x\right), n, x \in N, \Phi \in \mathcal{P}_{\omega \mid N}(N)$, i.e., we have the relation

$$
\langle\lambda(n) f, \Phi\rangle=\left\langle f, \lambda^{*}\left(n^{-1}\right) \Phi\right\rangle .
$$

Likewise

$$
\left(\rho^{*}(n) \Phi\right)(x)=\chi_{p}(n) \Phi(x n) .
$$

Taking annihilators with respect to the pairing $\mathcal{A} \times \mathcal{P}_{\omega \mid N}(N) \rightarrow \mathbb{C}$, the (proper) two-sided ideals in $\mathcal{A}$ correspond, in an antitone manner, to the (non-zero) $N$ biinvariant subspaces of $\mathcal{P}_{\omega \mid N}(N)$. For example, $\mathfrak{m}=\operatorname{ker}\left(\chi_{p}\right) / j\left(\chi_{p}\right)$ corresponds to the space of constant functions.

Conjugation by elements in $G$ induces an action $\kappa$ of $G$ on $\mathcal{A}$. Here the dual action $\kappa^{*}$ on $\mathcal{P}_{\omega \mid N}(N)$ is again conjugation, i.e., $\left(\kappa^{*}(a) \Phi\right)(x)=\Phi\left(a^{-1} x a\right), a \in G$, $x \in N, \Phi \in \mathcal{P}_{\omega \mid N}(N)$; observe that $\chi_{p}$ is invariant under conjugation. Hence the $G$-invariant ideals in $\mathcal{A}$ correspond to $N$-bi-invariant, $G$-invariant subspaces of $\mathcal{P}_{\omega \mid N}(N)$.

Consider two-sided ideals $\mathfrak{a}$ and $\mathfrak{b}$ in $\mathcal{A}$ such that $\mathfrak{b} \subset \mathfrak{a} \subset \mathfrak{m}$ and $\operatorname{dim}(\mathfrak{a} / \mathfrak{b})=1$ (for instance two consecutive members of the above Jordan-Hölder sequence). Then $\mathfrak{a} / \mathfrak{b}$ is an $\mathcal{A}$ - $\left(\right.$ or $L_{\omega \mid N}^{1}(N)$-) bimodule. As the maximal ideal $\mathfrak{m}\left(\right.$ or $\left.\operatorname{ker}\left(\chi_{p}\right)\right)$ annihilates $\mathfrak{a} / \mathfrak{b}$, we find that

$$
u f \equiv f u \equiv \chi_{p}(f) u \bmod \mathfrak{b}
$$

for $u \in \mathfrak{a}, f \in \mathcal{A}$ (or $\left.f \in L_{\omega \mid N}^{1}(N)\right)$. From this we conclude that the action of $x \in N$ on $\mathfrak{a} / \mathfrak{b}$, induced from $\lambda$, is given by multiplication with $\chi_{p}(x)$. If, in addition, $\mathfrak{a}$ and $\mathfrak{b}$ are $G$-invariant (under the conjugation $\kappa$ ), then the induced $G$-action on $\mathfrak{a} / \mathfrak{b}$ is trivial.

Moreover, there exist Jordan-Hölder sequences $\mathfrak{a}_{d}, \cdots, \mathfrak{a}_{1}, \mathfrak{a}_{0}$ in $\mathcal{A}$ with the additional property that the $\mathfrak{a}_{j}$ 's are $G$-invariant. If necessary one can realize (additionally) that the $\mathfrak{a}_{j}$ 's are invariant under the involution on $\mathcal{A}$, induced from the involution on $L_{\omega}^{1}(G)$. 
Convention 7.3. In the sequel, $\mathfrak{a}_{d} \supset \mathfrak{a}_{d-1}=\mathfrak{m} \supset \cdots \supset \mathfrak{a}_{1} \supset \mathfrak{a}_{0}$ will always denote a Jordan-Hölder sequence with all these properties.

The dense embedding $\mathcal{S}(N) \rightarrow L_{\omega \mid N}^{1}(N)$ induces a map from $\mathcal{S}(N)$ onto the finite-dimensional space $\mathcal{A}=L_{\omega \mid N}^{1}(N) / j\left(\chi_{p}\right)$. Hence $\mathcal{A}$ is canonically isomorphic to the algebra $\mathcal{S}(N) / \mathcal{S}(N) \cap j\left(\chi_{p}\right)$.

The closed two-sided ideals in $L_{\omega}^{1}(G)$ containing the minimal ideal $j\left(\pi_{l}\right)$, which is extended from $j\left(\chi_{p}\right) \subset L_{\omega \mid N}^{1}(N)$, correspond to the closed two-sided ideals in the quotient algebra $L_{\omega}^{1}(G) / j\left(\pi_{l}\right)$. The embedding $\mathcal{S}(G) \rightarrow L_{\omega}^{1}(G)$ induces a dense embedding $\mathcal{S}(G) / \mathcal{S}(G) \cap j\left(\pi_{l}\right) \rightarrow L_{\omega}^{1}(G) / j\left(\pi_{l}\right)$. To describe the quotient $\mathcal{S}(G) / \mathcal{S}(G) \cap j\left(\pi_{l}\right)$ it is convenient to view $\mathcal{S}(G)$ as a twisted covariance algebra. For the following see also Lue-Po, where the case of $L^{1}$-algebras is treated in a similar fashion. Given the normal subgroup $N$ of $G$ define the twist $l$ of $N\left(l=\right.$ left translation) on $\mathcal{S}(N)$ by $(l(n) f)(x)=f\left(n^{-1} x\right)$, and the action $c$ of $G$ on $\mathcal{S}(N)$ by $\left(c_{a} f\right)(x)=f\left(a^{-1} x a\right)$. Then one can form the twisted covariance algebra $\mathcal{S}(G / N, \mathcal{S}(N), c, l)$, or $\mathcal{S}(G / N, l)$ for short, which consists of functions $\varphi: G \rightarrow \mathcal{S}(N)$ such that

$$
\varphi(a x)=l(x)^{-1} \varphi(a), a \in G, x \in N,
$$

and that $\varphi$ is a Schwartz function on an appropriate cross section $S$ of $G \rightarrow G / N$.

The multiplication is given by

$$
(\varphi * \psi)(a)=\int_{G / N} c_{b}\left(\varphi\left(a b^{-1}\right)\right) * \psi(b) d \dot{b},
$$

where the "*" on the right hand side means convolution in $\mathcal{S}(N)$. Observe that the integrand, a function of $b \in G$, is in fact constant on $N$-cosets. The involution is given by

$$
\varphi^{*}(a)=c_{a^{-1}}\left(\varphi\left(a^{-1}\right)^{*}\right) .
$$

So far, this is just a complicated way to write the algebra $\mathcal{S}(G)$. Actually, if for $f \in \mathcal{S}(G)$ we define $\tilde{f}: G \rightarrow \mathcal{S}(N)$ by $\tilde{f}(a)(x)=f(a x)$, then the map $f \mapsto \tilde{f}$ yields an isomorphism from $\mathcal{S}(G)$ onto $\mathcal{S}(G / N, \mathcal{S}(N), c, l)$. The ideal $j\left(\pi_{l}\right) \cap \mathcal{S}(G)$ consists exactly of those $f \in \mathcal{S}(G)$ such that $\tilde{f}(x) \in j\left(\chi_{p}\right) \cap \mathcal{S}(N)$ for all $x \in G$, i.e., to the subspace $\mathcal{S}\left(G / N, j\left(\chi_{p}\right) \cap \mathcal{S}(N), c, l\right)$ of $\mathcal{S}(G / N, \mathcal{S}(N), c, l)$. Hence the quotient $\mathcal{S}(G) / \mathcal{S}(G) \cap j\left(\pi_{l}\right)$ - and this is the advantage of this approach - can be realized as the twisted covariance algebra

$$
\mathcal{S}(G / N, \mathcal{A}, c, \tau) \text {, or simply } \mathcal{S}(G / N, \tau) .
$$

Here $\mathcal{A}=\mathcal{S}(N) / \mathcal{S}(N) \cap j\left(\chi_{p}\right)$, the action $c$ of $G$ is the action induced by $c$ on $\mathcal{S}(N)$, while the twist $\tau$ on $\mathcal{A}$ is induced by left translation with elements in $N$. The multiplication and the involution on $\mathcal{S}(G / N, \tau)$ are given by the above formulae. As a Fréchet space, $\mathcal{S}(G / N, \tau)$ can be identified with the tensor product $\mathcal{S}(G / N) \otimes \mathcal{A}$.

The product $G \times G$ acts on $\mathcal{S}(G)$ via translations, $(\beta(a, b) f)(x)=f\left(a^{-1} x b\right)$ for $a, b, x \in G, f \in \mathcal{S}(G)$. Under the identification with $\mathcal{S}(G / N, \mathcal{S}(N), c, l)$, this action transforms into

$$
(\beta(a, b) \varphi)(x)=c_{b}\left(\varphi\left(a^{-1} x b\right)\right) .
$$

If $I$ is any Fréchet closed two-sided $G$-invariant ideal in $\mathcal{S}(N)$, for instance $I=$ $\mathcal{S}(N) \cap j\left(\chi_{p}\right)$, then this action yields an action of $G \times G$, denoted by the same letter $\beta$, on the quotient $\mathcal{S}(G / N, \mathcal{S}(N) / I, c, \tau)$ which is given by the above formula, where, 
of course, the above " $c_{b}$ " has to be replaced by the appropriate $c_{b}$, i.e., the induced action of $G$ of $\mathcal{S}(N) / I$. All these actions of $G \times G$ integrate to representations of the Schwartz algebra $\mathcal{S}(G \times G)$ on $G \times G$, also denoted by $\beta$.

The easiest way to construct ideals in $\mathcal{S}(G / N, \tau)$ is to take a $G$-invariant ideal $\mathfrak{a}$ in $\mathcal{A}$, i.e. $\mathfrak{a}=I / \mathcal{S}(N) \cap j\left(\chi_{p}\right)$ for some $G$-invariant two-sided ideal $I$ in $\mathcal{S}(N)$, closed and of finite codimension, and to form the extended ideal, which in the present approach means to form the collection $\mathcal{S}(G / N, \mathfrak{a}, c, \tau)$ of all $f \in \mathcal{S}(G / N, \mathcal{A}, c, \tau)$ such that $f(x) \in \mathfrak{a}$ for all $x \in G$. If $\mathfrak{b}$ is another $G$-invariant ideal in $\mathcal{A}$ such that $\mathfrak{b} \subset \mathfrak{a} \subset$ $\mathfrak{m}$ and $\operatorname{dim}(\mathfrak{a} / \mathfrak{b})=1$, then, as we remarked earlier, the action $c$ of $G$ on $\mathfrak{a} / \mathfrak{b}$ is trivial and the twist $\tau$ of $N$ on $\mathfrak{a} / \mathfrak{b}$ is multiplication by $\chi_{p}$. Identifying $\mathfrak{a} / \mathfrak{b}$ with $\mathbb{C}$ we find $\mathcal{S}(G / N, \mathfrak{a}, c, \tau) / \mathcal{S}(G / N, \mathfrak{b}, c, \tau) \cong \mathcal{S}(G / N, \mathfrak{a} / \mathfrak{b}, c, \tau) \cong \mathcal{S}\left(G / N, \chi_{p}\right)$, where (cf. section (4) $\mathcal{S}\left(G / N, \chi_{p}\right)$ consists of all functions $f: G \rightarrow \mathbb{C}$ with $f(a x)=\chi_{p}(x)^{-1} f(a)$, $a \in G, x \in N$, and $f$ is a "Schwartz function modulo $N$ ". The space $\mathcal{S}\left(G / N, \chi_{p}\right)$ is also an algebra, but the above identification is not an isomorphism of algebras: The multiplication on $\mathcal{S}(G / N, \mathfrak{a} / \mathfrak{b}, c, \tau)$ is identically zero (as the multiplication on $\mathfrak{a} / \mathfrak{b}$ is trivial). However, if we replace $\mathfrak{a}$ by $\mathcal{A}$ and $\mathfrak{b}$ by $\mathfrak{m}$, then it is still true that $\mathcal{S}(G / N, \mathcal{A} / \mathfrak{m}, c, \tau)$ is isomorphic to $\mathcal{S}\left(G / N, \chi_{p}\right)$, and this is an isomorphism of algebras. As the actions of $\mathfrak{m}$ on $\mathfrak{a} / \mathfrak{b}$ are trivial (from both sides), we observe the following remark.

Remark 7.4. The actions of $\mathcal{S}(G / N, \mathcal{A}, c, \tau)$ on $\mathcal{S}(G / N, \mathfrak{a} / \mathfrak{b}, c, \tau)$ reduce to actions of the quotient $\mathcal{S}(G / N, \mathcal{A} / \mathfrak{m}, c, \tau)$. Identifying, as above, the spaces $\mathcal{S}(G / N, \mathfrak{a} / \mathfrak{b}, c, \tau)$ and $\mathcal{S}(G / N, \mathcal{A} / \mathfrak{m}, c, \tau)$ with $\mathcal{S}\left(G / N, \chi_{p}\right)$, these actions yield the multiplication on the algebra $\mathcal{S}\left(G / N, \chi_{p}\right)$.

A (Fréchet-)closed subspace of $\mathcal{S}(G / N, \mathcal{A}, c, \tau)$ is a two-sided ideal if and only if it is invariant under $\beta(G \times G)$ or under $\beta(\mathcal{S}(G \times G))$. It turns out that in fact any $\beta(\mathcal{S}(G \times G))$-invariant subspace of $\mathcal{S}(G / N, \mathcal{A}, c, \tau)$ is automatically closed.

Proposition 7.5. Each $\mathcal{S}(G \times G)$-submodule $\mathcal{U}$ of $\mathcal{S}(G / N, \tau)$ is closed.

Proof. Recall, from Convention 7.3, that $0=\mathfrak{a}_{0} \subset \mathfrak{a}_{1} \subset \cdots \subset \mathfrak{m}=\mathfrak{a}_{d-1} \subset \mathfrak{a}_{d}=\mathcal{A}$ is a Jordan-Hölder sequence consisting of (self-adjoint) $G$-invariant two-sided ideals in $\mathcal{A}$. We proceed by finite induction on $k=\operatorname{dim}\left(\mathfrak{a}_{k}\right)$. Suppose first that $\mathcal{U}$ is contained in $\mathcal{S}\left(G / N, \mathfrak{a}_{1}, c, \tau\right)$, which is isomorphic to $\mathcal{S}\left(G / N, \chi_{p}\right)$. As $c$ is trivial the action of $G$ is given just by ordinary translations, $(\beta(a, b) f)(x)=f\left(a^{-1} x b\right)$, and by Proposition $4.1 \mathcal{S}\left(G / N, \chi_{p}\right)$ is a simple $\mathcal{S}(G \times G)$-module. Therefore, $\mathcal{U}=0$ or $\mathcal{U}=\mathcal{S}\left(G / N, \chi_{p}\right)$. Now suppose that $\mathcal{U}$ is contained in $\mathcal{S}\left(G / N, \mathfrak{a}_{k+1}, c, \tau\right)$, but not in $\mathcal{S}\left(G / N, \mathfrak{a}_{k}, c, \tau\right)$. By induction, we know that $\mathcal{U} \cap \mathcal{S}\left(G / N, \mathfrak{a}_{k}, c, \tau\right)$ is closed. Consider the exact sequence

$$
0 \rightarrow \mathcal{S}\left(G / N, \mathfrak{a}_{k}, c, \tau\right) \rightarrow \mathcal{S}\left(G / N, \mathfrak{a}_{k+1}, c, \tau\right) \stackrel{\nu}{\rightarrow} \mathcal{S}\left(G / N, \mathfrak{a}_{k+1} / \mathfrak{a}_{k}, c, \tau\right) \rightarrow 0 .
$$

The image $\nu(\mathcal{U})$ is a non-zero $\mathcal{S}(G \times G)$-submodule of $\mathcal{S}\left(G / N, \mathfrak{a}_{k+1} / \mathfrak{a}_{k}, c, \tau\right) \cong$ $\mathcal{S}\left(G / N, \chi_{p}\right)$. Hence $\nu$ maps $\mathcal{U}$ onto the latter space.

Choose any $u_{0} \in \mathcal{U}$ such that $\nu\left(u_{0}\right) \neq 0$. This element $\nu\left(u_{0}\right)$ is used as a "generator" of $\mathcal{S}\left(G / N, \mathfrak{a}_{k+1} / \mathfrak{a}_{k}, c, \tau\right)$, i.e., the map

$$
\mathcal{S}(G \times G) \rightarrow \mathcal{S}\left(G / N, \mathfrak{a}_{k+1} / \mathfrak{a}_{k}, c, \tau\right) ;
$$

$\varphi \mapsto \beta(\varphi)\left(\nu\left(u_{0}\right)\right)$ is surjective (and open). Let $\left(u_{j}\right)$ be any sequence in $\mathcal{U}$ with limit $f$ in $\mathcal{S}\left(G / N, \mathfrak{a}_{k+1}, c, \tau\right)$. We write $\nu\left(u_{j}\right)=\beta\left(\varphi_{j}\right)\left(\nu\left(u_{0}\right)\right), \nu(f)=\beta(\varphi)\left(\nu\left(u_{0}\right)\right)$ 
with $\varphi_{j}, \varphi \in \mathcal{S}(G \times G)$, and we may assume that $\left(\varphi_{j}\right)$ converges to $\varphi$. As $\nu\left(u_{j}\right)=$ $\nu\left(\beta\left(\varphi_{j}\right) u_{0}\right)$ we see that $u_{j}-\beta\left(\varphi_{j}\right) u_{0} \in \mathcal{U} \cap \mathcal{S}\left(G / N, \mathfrak{a}_{k}, c, \tau\right)$. Therefore, the limit of this sequence, namely $f-\beta(\varphi) u_{0}$, is in $\mathcal{U}$, which shows $f \in \mathcal{U}$.

Among others we will show later that each $\mathcal{S}(G \times G)$-submodule of $\mathcal{S}(G / N, \tau)$ is finitely generated (with at most $d=\operatorname{dim}(\mathcal{A})$ generators). To this end, we use idempotents in the algebra $\mathcal{S}(G / N, \tau)$ whose existence is established first.

Lemma 7.6. Let $q$ be a projection of rank 1 in the algebra $\mathcal{S}\left(G / N, \chi_{p}\right)$, i.e., $q^{*}=q$, $q * q=q, q * \mathcal{S}\left(G / N, \chi_{p}\right) * q=\mathbb{C} q \neq 0$. There exists a $\mathfrak{q} \in \mathcal{S}(G / N, \tau)$ such that $\mathfrak{q}^{*}=\mathfrak{q}, \mathfrak{q} * \mathfrak{q}=\mathfrak{q}, \mu(\mathfrak{q})=q$, where $\mu$ denotes the canonical map

$$
\mathcal{S}(G / N, \mathcal{A}, c, \tau) \stackrel{\mu}{\rightarrow} \mathcal{S}\left(G / N, \mathcal{S}(N) / \operatorname{ker}\left(\chi_{p}\right)\right)=\mathcal{S}(G / N, \mathcal{A} / \mathfrak{m}, c, \tau) \cong \mathcal{S}\left(G / N, \chi_{p}\right) .
$$

Proof. First we note that there are plenty of such q's. By [Ho], to each smooth vector $\xi \neq 0$ for the representation $\pi_{l}$ there exists $f=f^{*} \in \mathcal{S}(G)$ such that $\pi_{l}(f)$ is the orthogonal projection onto $\mathbb{C} \xi$. Let $q \in \mathcal{S}\left(G / N, \chi_{p}\right)$ be the element corresponding to $\pi_{l}(f)$ under the canonical identification $\mathcal{S}\left(G / N, \chi_{p}\right) \cong \pi_{l}(\mathcal{S}(G))$.

Given $q$ choose any $h=h^{*}$ in $\mathcal{S}(G / N, \tau)$, which is mapped onto $q$, for instance the image of the above $f$ under the canonical surjection $\mathcal{S}(G) \rightarrow \mathcal{S}(G / N, \tau)$. Since $h(1-h) \in \operatorname{ker}(\mu), \operatorname{ker}(\mu) \cong \mathcal{S}(G) \cap \operatorname{ker}\left(\pi_{l}\right) / \mathcal{S}(G) \cap j\left(\pi_{l}\right)$, and $\left(\operatorname{ker}\left(\pi_{l}\right)\right)^{d} \subset j\left(\pi_{l}\right)$ by Remark 3.5, it follows that $0=(h(1-h))^{d}=h^{d}(1-h)^{d}$.

The polynomials $t^{d}$ and $(1-t)^{d}$ are relatively prime in $\mathbb{Q}[t]$. Hence there exist $\Phi, \Psi \in \mathbb{Q}[t]$ with $1=t^{d} \Phi(t)+(1-t)^{d} \Psi(t)$. Actually, a possible pair $\Phi, \Psi$ can be constructed explicitly: From

$$
\begin{aligned}
1 & =(t+(1-t))^{2 d-1} \\
& =\sum_{k=d}^{2 d-1}\left(\begin{array}{c}
2 d-1 \\
k
\end{array}\right) t^{k}(1-t)^{2 d-1-k}+\sum_{k=0}^{d-1}\left(\begin{array}{c}
2 d-1 \\
k
\end{array}\right) t^{k}(1-t)^{2 d-1-k} \\
& =t^{d} \sum_{k=d}^{2 d-1}\left(\begin{array}{c}
2 d-1 \\
k
\end{array}\right) t^{k-d}(1-t)^{2 d-1-k}+(1-t)^{d} \sum_{k=0}^{d-1}\left(\begin{array}{c}
2 d-1 \\
k
\end{array}\right) t^{k}(1-t)^{d-1-k}
\end{aligned}
$$

one can read $\Phi$ and $\Psi$. In the quotient algebra $\mathbb{Q}[t] / t^{d}(1-t)^{d}$ the polynomials $t^{d} \Phi(t)$ and $\left(1-t^{d} \Psi(t)\right.$ yield idempotents $\left(\left(t^{d} \Psi(t)\right)\right)^{2}-t^{d} \Phi(t)=t^{d} \Phi(t)\left(t^{d} \Psi(t)-1\right)=$ $\left.-t^{d} \Phi(t)(1-t)^{d} \Psi(t) \equiv 0 \bmod t^{d}(1-t)^{d}\right)$. As the homomorphism $\mathbb{Q}[t] \ni F \mapsto F(h) \in$ $\mathcal{S}(G / N, \tau)$ factors through $\mathbb{Q}[t] / t^{d}(1-t)^{d}$, the element $\mathfrak{q}:=h^{d} \Phi(h)$ of $\mathcal{S}(G / N, \tau)$ is idempotent as well. Also the other two claimed properties of $\mathfrak{q}$ are satisfied. Since $t^{d} \Phi(t)$ has real coefficients, $\mathfrak{q}$ is selfadjoint. Finally, from $1=t^{d} \Phi(t)+(1-t)^{d} \Psi(t)$ we conclude $\Phi(1)=1$, i.e., $\sum_{j \geq 0} \varphi_{j}=1$ if $\Phi(t)=\sum_{j \geq 0} \varphi_{j} t^{j}$. Hence $\mu(\mathfrak{q})=\mu\left(h^{d} \Phi(h)\right)=$ $q^{d} \sum_{j \geq 0} \varphi_{j} q^{j}=\left(\sum_{j \geq 0} \varphi_{j}\right) q=q$

An idempotent $\mathfrak{q}$ as in the lemma is fixed for a while. We consider the evaluation map $E v$ at the unit element in $G$ for functions $\varphi \in \mathcal{S}(G / N, \mathcal{A}, c, \tau)$, and for subquotients $\mathcal{S}\left(G / N, \mathfrak{a}_{k+1} / \mathfrak{a}_{k}, c, \tau\right)$ as well.

Proposition 7.7. $E v: \mathfrak{q} * \mathcal{S}(G / N, \mathcal{A}, c, \tau) * \mathfrak{q} \rightarrow \mathcal{A}$ is a linear isomorphism; in particular, $\operatorname{dim}(\mathfrak{q} * \mathcal{S}(G / N, \mathcal{A}, c, \tau) * \mathfrak{q})=d$. 
Proof. By induction we prove that $E v$ gives a linear isomorphism from $\mathfrak{q} * \mathcal{S}\left(G / N, \mathfrak{a}_{k}, c, \tau\right) * \mathfrak{q}$ onto $\mathfrak{a}_{k}$. For $k=1$ we have, as before, $\mathcal{S}\left(G / N, \mathfrak{a}_{1}, c, \tau\right) \cong$ $\mathcal{S}\left(G / N, \chi_{p}\right)$, and the action of $\mathcal{S}(G / N, \tau)$ leads (cf. Remark 7.4) to the action of $\mathcal{S}\left(G / N, \chi_{p}\right)$ from both sides. Hence we find that $\mathfrak{q} * \mathcal{S}\left(G / N, \mathfrak{a}_{1}, c, \tau\right) * \mathfrak{q}$ can be identified as $q * \mathcal{S}\left(G / N, \chi_{p}\right) * q=\mathbb{C} q$. It remains to observe that $q(e)=$ $\int_{G / N} q(x) q\left(x^{-1}\right) d x=\int_{G / N} q(x) \overline{q(x)} d \dot{x}$ is different from zero.

For the induction step consider the commutative diagram

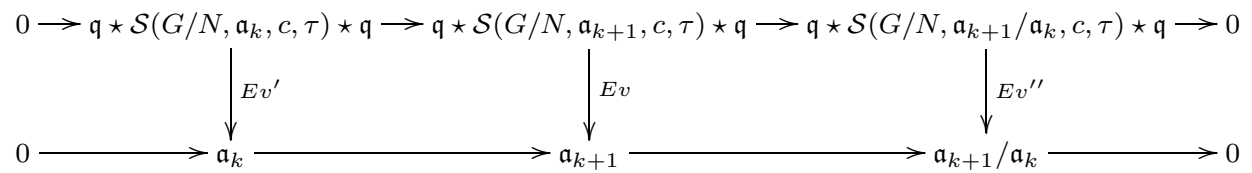

with exact lines where $E v, E v^{\prime}$, and $E v^{\prime \prime}$ denote the various evaluation maps at the unit in $G$. The map $E v^{\prime}$ is a linear isomorphism by induction. As above, in the case $k=1$, one sees that $E v^{\prime \prime}$ is a linear isomorphism between these one-dimensional spaces. Therefore, $E v$ has to be a linear isomorphism.

Remark 7.8. For any $k, j, 0 \leq k<j \leq d$, one also has a linear isomorphism

$$
R: \mathfrak{a}_{j} / \mathfrak{a}_{k} \rightarrow \mathfrak{q} * \mathcal{S}\left(G / N, \mathfrak{a}_{j} / \mathfrak{a}_{k}, c, \tau\right) * \mathfrak{q}
$$

given by

$$
\mathfrak{a}_{j} / \mathfrak{a}_{k} \ni u \mapsto f_{u}, f_{u}(x)=\int_{G / N} c_{y}\{\mathfrak{q}(x y) * u\} * \mathfrak{q}\left(y^{-1}\right) d \dot{y} .
$$

The proof for this fact proceeds along the same lines as the previous one.

Next we consider the closed two-sided ideals in $\mathcal{S}(G / N, \mathcal{A}, c, \tau)$. Recall that the latter algebra is isomorphic to $\mathcal{S}(G) / \mathcal{S}(G) \cap j\left(\pi_{l}\right)$ so that those ideals correspond to the closed two-sided ideals of $\mathcal{S}(G)$ containing $\mathcal{S}(G) \cap j\left(\pi_{l}\right)$. For the special ideals $\mathcal{S}\left(G / N, \mathfrak{a}_{k}, c, \tau\right)$ we first observe the following

Lemma 7.9. For each $k, 1 \leq k \leq d, \mathcal{S}(G / N, \mathcal{A}, c, \tau) *\left(\mathfrak{q} * \mathcal{S}\left(G / N, \mathfrak{a}_{k}, c, \tau\right) * \mathfrak{q}\right) *$ $\mathcal{S}(G / N, \mathcal{A}, c, \tau)$ is total in $\mathcal{S}(G / N, \tau)$, i.e., the $\mathcal{S}(G \times G)$-module, for the action $\beta$ (see above), generated by $\mathfrak{q} * \mathcal{S}\left(G / N, \mathfrak{a}_{k}, c, \tau\right) * \mathfrak{q}$ is equal to $\mathcal{S}\left(G / N, \mathfrak{a}_{k}, c, \tau\right)$. In particular $\mathfrak{q} * \mathcal{S}(G / N, \mathcal{A}, c, \tau) * \mathfrak{q}$ generates the whole space $\mathcal{S}(G / N, \tau)$.

Proof. The easy proof (by induction) follows the usual lines and is omitted.

Theorem 7.10. Any $\mathcal{S}(G \times G)$-submodule $J$ of $\mathcal{S}(G / N, \mathcal{A}, c, \tau)$, i.e., any closed two-sided ideal, is finitely generated. Actually, it is generated (in an algebraic sense) by $\mathfrak{q} * J * \mathfrak{q}$, whose dimension doesn't exceed $d ; c f$. Proposition 7.7. The map $J \mapsto \mathfrak{q} * J * \mathfrak{q}$ is an inclusion preserving bijection from the set of closed two-sided ideals in $\mathcal{S}(G / N, \tau)$ onto the set of two-sided ideals in the finite-dimensional algebra $\mathfrak{q} * \mathcal{S}(G / N, \mathcal{A}, c, \tau) * \mathfrak{q}$.

Proof. Write $\mathcal{S}=\mathcal{S}(G / N, \mathcal{A}, c, \tau)$ for short. As $\mathcal{S} *(\mathfrak{q} * J * \mathfrak{q}) * \mathcal{S} \supset(\mathcal{S} *(\mathfrak{q} * \mathcal{S} *$ $\mathfrak{q}) * \mathcal{S}) * J *(\mathcal{S} *(\mathfrak{q} * \mathcal{S} * \mathfrak{q}) * \mathcal{S})$, and $\mathcal{S} *(\mathfrak{q} * \mathcal{S} * \mathfrak{q}) * \mathcal{S}$ is total in $\mathcal{S}$ by the previous lemma, we conclude that $\mathcal{S} *(\mathfrak{q} * J * \mathfrak{q}) * \mathcal{S}$ is total in $J$. The rest of the theorem follows more or less immediately. 
Above, in Proposition 7.7 and Remark 7.8 we have seen two linear isomorphisms, $E v$ and $R$, between $\mathfrak{q} * \mathcal{S}(G / N, \mathcal{A}, c, \tau) * \mathfrak{q}$ and $\mathcal{A}$. In the case where $J$ is an extended ideal $J=\mathcal{S}(G / N, \mathfrak{a}, c, \tau)$ for some two-sided $G$-invariant ideal $\mathfrak{a}$ in $\mathcal{A}$, it is natural to ask how $R$ and $E v$ relate $\mathfrak{q} * J * \mathfrak{q}$ to $\mathfrak{a}$. One obtains the expected answer.

Remark 7.11. One has $R(\mathfrak{a})=\mathfrak{q} * J * \mathfrak{q}$ and $E v(\mathfrak{q} * J * \mathfrak{q})=\mathfrak{a}$.

Proof. Clearly, the functions in $R(\mathfrak{a})$ take their values in $\mathfrak{a}$; thus $R(\mathfrak{a}) \subset J \cap \mathfrak{q} *$ $\mathcal{S}(G / N, \mathcal{A}, c, \tau) * \mathfrak{q}=\mathfrak{q} * J * \mathfrak{q}$. On the other hand, $E v(\mathfrak{q} * J * \mathfrak{q}) \subset \mathfrak{a}$. As $R$ and $E v$ are linear isomorphisms, for dimensional reasons the above inclusions have to be equalities.

For the transition to the ideal theory in $\mathcal{B}=L_{\omega}^{1}(G) / j\left(\pi_{l}\right)$ it is convenient to view $S(G / N, \mathcal{A}, c, \tau) \cong \mathcal{S}(G) / \mathcal{S}(G) \cap j\left(\pi_{l}\right)$ as a dense subalgebra, say $\mathcal{B}_{\mathcal{S}}$ of $\mathcal{B}$. From the previous discussion we observe two facts:

(i) $\mathfrak{q} * \mathcal{B} * \mathfrak{q}=\mathfrak{q} * \mathcal{B}_{\mathcal{S}} * \mathfrak{q} \subset \mathcal{B}_{\mathcal{S}}$ (as $\mathcal{B}_{S}$ is dense in $\mathcal{B}$ and $\mathfrak{q} * \mathcal{B}_{\mathcal{S}} * \mathfrak{q}$ is finitedimensional); in particular, $\mathfrak{q} * J * \mathfrak{q} \subset \mathfrak{q} * \mathcal{B}_{\mathcal{S}} * \mathfrak{q}$ for any closed two-sided ideal $J$ in $\mathcal{B}$.

(ii) $\mathcal{B}_{\mathcal{S}} * \mathfrak{q} * \mathcal{B}_{\mathcal{S}} * \mathfrak{q} * \mathcal{B}_{\mathcal{S}}$ is total in $\mathcal{B}$.

Using these facts one easily concludes.

Theorem 7.12. The map $J \mapsto \mathfrak{q} * J * \mathfrak{q} \subset \mathfrak{q} * \mathcal{B}_{\mathcal{S}} * \mathfrak{q}$ establishes an order preserving bijection from the set of closed two-sided ideals in $\mathcal{B}=L_{\omega}^{1}(G) / j\left(\pi_{l}\right)$, i.e., the set of closed two-sided ideals in $L_{\omega}^{1}(G)$ containing $j\left(\pi_{l}\right)$, onto the set of two-sided ideals in $\mathfrak{q} * \mathcal{B}_{\mathcal{S}} * \mathfrak{q} \cong \mathfrak{q} * \mathcal{S}(G / N, \mathcal{A}, c, \tau) * \mathfrak{q}$. In particular, any closed two-sided ideal $J$ in $\mathcal{B}$ is generated, as a closed two-sided ideal, by the finite-dimensional space $\mathfrak{q} * J * \mathfrak{q}$ sitting in $\mathcal{B}_{\mathcal{S}}$.

One would like to know more about the set of ideals in $\mathfrak{q} * \mathcal{S}(G / N, \mathcal{A}, c, \tau) * \mathfrak{q}$, or, even better, to "know" this algebra itself. This $d$-dimensional algebra has a unit, namely q; moreover it has a Jordan-Hölder sequence

$$
\mathfrak{q} * \mathcal{S}\left(G / N, \mathfrak{a}_{k}, c, \tau\right) * \mathfrak{q}, 0 \leq k \leq d .
$$

Briefly, $\mathfrak{q} * \mathcal{S}(G / N, \mathcal{A}, c, \tau) * \mathfrak{q}$ has all the properties as described in Lemma 7.1 . Since the dimension of this algebra equals $\operatorname{dim}(\mathcal{A})$, it is plausible, at first glance, to guess that $\mathfrak{q} * \mathcal{S}(G / N, \mathcal{A}, c, \tau) * \mathfrak{q}$ is isomorphic (as an algebra) to $\mathcal{A}$. However, with a second glance, it is no longer plausible because $\mathfrak{q} * \mathcal{S}(G / N, \mathcal{A}, c, \tau) * \mathfrak{q}$ depends, at least implicitly, on the action $c$ of $G$ on $\mathcal{A}$, while the algebra $\mathcal{A}$ does not "know" this action. But, if $N$ is central in $G$ (compare section 6), which implies that $\mathcal{A}$ is commutative and that the action $c$ of $G$ on $\mathcal{A}$ is trivial, then in fact $\mathcal{A}$ is isomorphic to $\mathfrak{q} * \mathcal{S}(G / N, \mathcal{A}, c, \tau) * \mathfrak{q}$.

Proposition 7.13. If $N=G(l)$ is central in $G$, then the map

$$
R: \mathcal{A} \rightarrow \mathfrak{q} * \mathcal{S}(G / N, \mathcal{A}, c, \tau) * \mathfrak{q}
$$

of Remark 7.8, i.e.,

$$
(R u)(x)=\int_{G / N} c_{y}(\mathfrak{q}(x y) * u) * \mathfrak{q}\left(y^{-1}\right) d \dot{y},
$$

is an isomorphism of algebras. 
Proof. In the case at hand the map $R$ simplifies to $(R u)(x)=\mathfrak{q}(x) u$. One easily computes

$$
\begin{aligned}
(R u * R v)(x) & =\int_{G / N} R_{u}(x y) R_{v}\left(y^{-1}\right) d \dot{y} \\
& =\int_{G / N} \mathfrak{q}(x y) u \mathfrak{q}\left(y^{-1}\right) v d \dot{y}=\int_{G / N} \mathfrak{q}(x y) \mathfrak{q}\left(y^{-1}\right) u v d \dot{y} \\
& =\mathfrak{q}(x) u v \text { as } \mathfrak{q} \text { is an idempotent } \\
& =(R(u v))(x) .
\end{aligned}
$$

There are many choices for the self-adjoint idempotent $\mathfrak{q}$, and it is natural to ask to which extent the algebra $\mathfrak{q} * \mathcal{S}(G / N, \mathcal{A}, c, \tau) * \mathfrak{q}$ depends on this choice.

Proposition 7.14. Again write $\mathcal{S}=\mathcal{S}(G / N, \mathcal{A}, c, \tau)$ for short. Suppose that $\mathfrak{q}_{1}$ and $\mathfrak{q}_{2}$ are two self-adjoint idempotents in $\mathcal{S}$ such that, for the canonical map $\mu$ : $\mathcal{S} \rightarrow \mathcal{S}(G / N, \mathcal{A} / \mathfrak{m}, c, \tau) \cong \mathcal{S}\left(G / N, \chi_{p}\right)$, one has that $q_{j}:=\mu\left(\mathfrak{q}_{j}\right)$ is a projector of rank 1; cf. Lemma 7.6. Then there exists $\mathfrak{v} \in \mathfrak{q}_{1} * \mathcal{S} * \mathfrak{q}_{2}$ such that $\mathfrak{q}_{1}=\mathfrak{v} * \mathfrak{v}^{*}$, $\mathfrak{q}_{2}=\mathfrak{v}^{*} * \mathfrak{v}$. Moreover, the map $\mathfrak{q}_{1} * \mathcal{S} * \mathfrak{q}_{1} \rightarrow \mathfrak{q}_{2} * \mathcal{S} * \mathfrak{q}_{2}$, given by $f \mapsto \mathfrak{v}^{*} * f * \mathfrak{v}$, is an isomorphism of algebras.

Proof. Denote by $\mu_{k}, 1 \leq k \leq d$, the natural homomorphism

$$
\mathcal{S}=\mathcal{S}(G / N, \mathcal{A}, c, \tau) \rightarrow \mathcal{S}\left(G / N, \mathcal{A} / \mathfrak{a}_{d-k}, c, \tau\right),
$$

especially $\mu_{1}=\mu$. We prove, by induction on $k$, that there exists $\mathfrak{v}_{k} \in \mu_{k}\left(\mathfrak{q}_{1}\right) *$ $\mathcal{S}\left(G / N, \mathcal{A} / \mathfrak{a}_{d-k}, c, \tau\right) * \mu_{k}\left(\mathfrak{q}_{2}\right)$ such that $\mathfrak{v}_{k}^{*} * \mathfrak{v}_{k}=\mu_{k}\left(\mathfrak{q}_{2}\right), \mathfrak{v}_{k} * \mathfrak{v}_{k}^{*}=\mu_{k}\left(\mathfrak{q}_{1}\right)$. For $k=1$ take any non-zero $\mathfrak{w}$ in the one-dimensional space $q_{1} * \mathcal{S}(G / N, \mathcal{A} / \mathfrak{m}, c, \tau) * q_{2}$. Then one has $\mathfrak{w} * \mathfrak{w}^{*}=z_{1} q_{1}$ and $\mathfrak{w}^{*} * \mathfrak{w}=z_{2} q_{2}$ with some constants $z_{1}, z_{2}$. Applying the representation $\pi_{l}$ of $\mathcal{S}(G) / \operatorname{ker}\left(\pi_{l}\right) \cong \mathcal{S}(G / N, \mathcal{A} / \mathfrak{m}, c, \tau)$ one sees that $z_{1}=z_{2}$, and this number is positive. Denote this common constant by $z$ and put $\mathfrak{v}_{1}=\left(\frac{1}{z}\right)^{1 / 2} \mathfrak{w}$.

To proceed from $k, 1 \leq k<d$ to $k+1$, take $\mathfrak{v}_{k}$ as above and take any $\mathfrak{w}$ in $\mu_{k+1}\left(\mathfrak{q}_{1}\right) * \mathcal{S}\left(G / N, \mathcal{A} / \mathfrak{a}_{d-k-1}, c, \tau\right) * \mu_{k+1}\left(\mathfrak{q}_{2}\right)$ such that $\mathfrak{w}$ is mapped onto $\mathfrak{v}_{k}$ for the canonical map

$$
\mathcal{S}\left(G / N, \mathcal{A} / \mathfrak{a}_{d-k-1}, c, \tau\right) \rightarrow \mathcal{S}\left(G / N, \mathcal{A} / \mathfrak{a}_{d-k}, c, \tau\right) .
$$

Then one has

$$
\begin{aligned}
& \mathfrak{w}^{*} * \mathfrak{w}=\mu_{k+1}\left(\mathfrak{q}_{2}\right)+\varphi \\
& \mathfrak{w} * \mathfrak{w}^{*}=\mu_{k+1}\left(\mathfrak{q}_{1}\right)+\psi
\end{aligned}
$$

with some self-adjoint elements $\varphi$ and $\psi$ in the (one-dimensional) spaces

$$
\mu_{k+1}\left(\mathfrak{q}_{2}\right) * \mathcal{S}\left(G / N, \mathfrak{a}_{d-k} / \mathfrak{a}_{d-k-1}, c, \tau\right) * \mu_{k+1}\left(\mathfrak{q}_{2}\right)
$$

and $\mu_{k+1}\left(\mathfrak{q}_{1}\right) * \mathcal{S}\left(G / N, \mathfrak{a}_{d-k} / \mathfrak{a}_{d-k-1}, c, \tau\right) * \mu_{k+1}\left(\mathfrak{q}_{1}\right)$, respectively. From the identity $\mathfrak{w} *\left(\mathfrak{w}^{*} * \mathfrak{w}\right)=\left(\mathfrak{w} * \mathfrak{w}^{*}\right) * \mathfrak{w}$ one concludes

$$
\begin{aligned}
\mathfrak{w} *\left(\mu_{k+1}\left(\mathfrak{q}_{2}\right)+\varphi\right) & =\mathfrak{w}+\mathfrak{w} * \varphi \\
& =\left(\mu_{k+1}\left(\mathfrak{q}_{1}\right)+\psi\right) * \mathfrak{w} \\
& =\mathfrak{w}+\psi * \mathfrak{w},
\end{aligned}
$$

hence $\mathfrak{w} * \varphi=\psi * \mathfrak{w}$. 
Likewise the identity $\left(\mathfrak{w}^{*} * \mathfrak{w}\right) * \mathfrak{w}^{*}=\mathfrak{w}^{*} *\left(\mathfrak{w} * \mathfrak{w}^{*}\right)$ yields $\varphi * \mathfrak{w}^{*}=\mathfrak{w}^{*} * \psi$.

This equation also follows from the previous one by applying the involution and using $\varphi=\varphi^{*}, \psi=\psi^{*}$.

Now put $\mathfrak{v}_{k+1}=\mathfrak{w}-\frac{1}{2} \mathfrak{w} * \varphi=\mathfrak{w}-\frac{1}{2} \psi * \mathfrak{w}$. The above relations tell us that $\mathfrak{v}_{k+1}$ has the required properties. Finally $\mathfrak{v}:=\mathfrak{v}_{d}$ gives an element as claimed in the proposition. Moreover, it is easy to see that $f \mapsto \mathfrak{v}^{*} * f * \mathfrak{v}$ is an algebra homomorphism, the inverse being given by $g \mapsto \mathfrak{v} * g * \mathfrak{v}^{*}$.

\section{TWO EXAMPLES}

To build the following examples, we use the method developed in sections 5 and 6

\subsection{The step 2 case.}

8.1.1. Let $\mathfrak{g}=\operatorname{span}\{A, B, C, U, V, W\}$ be the free nilpotent step two Lie algebra with three generators, i.e. with the brackets

$$
[A, B]=U,[B, C]=V,[C, A]=W .
$$

Let $G=(\mathfrak{g}, \cdot)$ be the simply connected Lie group with Lie algebra $\mathfrak{g}$ equipped with the Baker-Campbell-Hausdorff multiplication. This means that

$$
X \cdot Y=X+Y+\frac{1}{2}[X, Y], X, Y \in \mathfrak{g} .
$$

We take coordinates of the first kind on $G$, i.e.

$$
a A+b B+c C+u U+v V+w W \simeq(a, b, c, u, v, w) .
$$

Let $\ell=U^{*}$. This gives us $\mathfrak{g}(\ell)=\mathfrak{n}=\operatorname{span}(C, U, V, W)$.

We take a weight $\omega$ on $G$ which allows only polynomials of degree one inside $\mathcal{P}_{\omega_{\mid N}}(N)$. One possibility is the following: We define $\omega$ on $G$ by $\omega(a, b, c, u, v, w):=$ $1+|a|+|c|+|w|$. Then $\left.\omega\right|_{N}(0,0, c, u, v, w)=1+|c|+|w|$. This shows that $\mathcal{P}_{\omega_{\mid N}}(N)=\operatorname{span}\left(p_{2}, p_{1}, 1\right)$ with $p_{1}(c, u, v, w)=c$ and $p_{2}(c, u, v, w)=w$. One checks that

$$
d \sigma(A) p_{2}=p_{1}, d \sigma(B) p_{2}=0 .
$$

Let $I$ be a two-sided closed ideal of $L_{\omega}^{1}(G)$ belonging to $\mathcal{I}^{\pi_{\ell}}$. As $G$ is step 2, $Y \cdot X \cdot Y=X+2 Y$ for all $X, Y \in G \equiv \mathfrak{g}$. Hence the ideal $I$ is invariant by abelian translation and by partial derivation, i.e. if $f \in I$, then $t(Y) f \in I$ where $t(Y) f$ is defined by $(t(Y) f)(X):=f(X+Y)$. Conversely, it is easy to check that a closed subset of $L_{\omega}^{1}(G)$ which is invariant by abelian translation and by usual conjugation in the group $G$ is also invariant by right and left translations in the group, i.e. is an ideal.

Let

$$
\varphi(a, b, c, u, v, w)=\left(\varphi_{2}(a, b) w+\varphi_{1}(a, b) c\right) e^{-i u}
$$

be a $C^{\infty}$-function in $I^{\perp}$. We may omit a possible term $\varphi_{0}(a, b) e^{-i u}$ in the expression of $\varphi$, as $\varphi_{0}(a, b) e^{-i u} \in \mathbb{C} \cdot \chi_{l}$ and as $I \subset \operatorname{ker}\left(\pi_{l}\right)=e_{G}\left(\operatorname{ker}\left(\chi_{l}\right)\right)$. Then for $f \in I^{\mathcal{S}}$, we have that

$$
\int_{G} \varphi^{(r A+s B)}(X) f(X) d X=\int_{G} \varphi(X+[r A+s B, X]) f(X) d X=0, r, s \in \mathbb{R},
$$


where $\varphi^{(r A+s B)}(X):=\varphi((r A+s B) \cdot X \cdot(-r A-s B))$. Hence, for $r, s \in \mathbb{R}$, $0=\int_{\mathbb{R}^{6}}\left(\varphi_{2}(a, b)(w-r c)+\varphi_{1}(a, b) c\right) e^{-i u} e^{-i(r b-s a)} f(a, b, c, u, v, w) d a d b d c d u d v d w$.

Therefore, by integration by parts,

$$
\begin{aligned}
0= & \int_{\mathbb{R}^{6}}\left(w \varphi_{2}(a, b)+c\left(\varphi_{1}(a, b)+i \partial_{2}\left(\varphi_{2}\right)(a, b)\right) f(a, b, c, u, v, w)\right. \\
& \left.+i c\left(\partial_{2}\right)(f)(a, b, c, u, v, w) \varphi_{2}(a, b)\right) e^{-i u} e^{-i(r b-s a)} d a d b d c d u d v d w
\end{aligned}
$$

where $\partial_{2}$ denotes the partial derivative with respect to the variable $b$. Hence, the Fourier inversion theorem implies that

$$
\begin{aligned}
0=\int_{\mathbb{R}^{4}}\left(w \varphi_{2}(a, b)+c\left(\varphi_{1}(a, b)+i \partial_{2} \varphi_{2}(a, b)\right) f(a, b, c, u, v, w)\right. \\
\left.\quad+i c \partial_{2}(f)(a, b, c, u, v, w) \varphi_{2}(a, b)\right) e^{-i u} d c d u d v d w, \quad a, b \in \mathbb{R} .
\end{aligned}
$$

As $f$ may be changed by arbitrary abelian translations (which commute with partial derivations), we even obtain

$$
\begin{aligned}
0=\int_{\mathbb{R}^{4}}\left(w \varphi_{2}\left(a_{1}, b_{1}\right)+c\left(\varphi_{1}\left(a_{1}, b_{1}\right)+i \partial_{2} \varphi_{2}\left(a_{1}, b_{1}\right)\right) f(a, b, c, u, v, w)\right. \\
\left.\quad+i c \partial_{2}(f)(a, b, c, u, v, w) \varphi_{2}\left(a_{1}, b_{1}\right)\right) e^{-i u} d c d u d v d w, \quad a, b, a_{1}, b_{1} \in \mathbb{R} .
\end{aligned}
$$

We denote in this subsection the mapping $\widetilde{\Psi}_{j}: \mathcal{S}(G) \rightarrow C^{\infty}(G)$ by

$$
\widetilde{\Psi}_{j}(f)(x):=\int_{N} f(x+n) p_{j}(n) \chi_{\ell}(n) d n, x \in G .
$$

One checks that for $f \in \operatorname{Ker} \pi_{l} \cap \mathcal{S}(G)$ and for $n \in N$,

$$
\widetilde{\Psi}_{j}(f)(x+n)=\overline{\chi_{l}(n)} \widetilde{\Psi}_{j}(f)(x), j=1,2, x \in G .
$$

Moreover,

$$
\operatorname{Ker} \widetilde{\Psi}_{1} \cap \operatorname{Ker} \widetilde{\Psi}_{2} \cap \operatorname{Ker} \pi_{l}=j\left(\pi_{l}\right) \cap \mathcal{S}(G) .
$$

Let us now take $\delta_{i} \in \mathcal{S}(N), i=1,2$, such that

$$
\int_{N} \delta_{j}(n) \chi_{\ell}(n) d n=0, \int_{N} p_{i}(n) \delta_{j}(n) \chi_{\ell}(n) d n=\delta_{i j}, i, j=1,2 .
$$

Let us consider functions of the form $f=h_{1} \otimes \delta_{1}+h_{2} \otimes \delta_{2}$ with $h_{1}, h_{2} \in \mathcal{S}\left(\mathbb{R}^{2}\right)$ arbitrary. Then

$$
\left.\int_{N} f\right|_{N}(n) \chi_{l}(n) d n=0
$$

by choice of $\delta_{1}, \delta_{2}$. So $\left.f\right|_{N} \in \operatorname{Ker} \chi_{\left.l\right|_{\mathfrak{n}}}$, and hence $f \in \operatorname{Ker} \pi_{l}$. We then have

$$
\widetilde{\Psi}_{j}(f)(s+n)=\overline{\chi_{l}(n)} \widetilde{\Psi}_{j}(f)(s)=\overline{\chi_{l}(n)} h_{j}(s), j=1,2 .
$$

Now let $I \in \mathcal{I}^{\pi_{\ell}}$ such that $j\left(\pi_{l}\right) \subsetneq I \subsetneq \operatorname{Ker} \pi_{l}$ and let us assume that $I$ is not $L^{\infty}(G / N)$-invariant. By Theorem 5.5 and by considerations on the dimensions, we have $\iota^{I}=\{1\}$ and $I^{\infty}=\langle f\rangle+j\left(\pi_{l}\right)^{\infty}$ for some $f \in \mathcal{S}(G)$. By Proposition 5.2, $f$ may be written in the form

$$
f=h_{1} \otimes \delta_{1}+h_{2} \otimes \delta_{2}
$$

for some $h_{1}, h_{2} \in \mathcal{S}\left(\mathbb{R}^{2}\right)$.

We now have to distinguish two cases. 
8.1.2. First case: Let $\varphi \in I^{\perp}$ be of the form

$$
\varphi(a, b, c, u, v, w)=\varphi_{1}(a, b) c e^{-i u}, a, b \in \mathbb{R},
$$

with $\varphi_{1} \not \equiv 0$, i.e. let us assume that $\varphi_{2} \equiv 0$. Then, for all $f \in I^{S}$,

$$
0=\varphi_{1}(a, b) \int_{\mathbb{R}^{4}} c f(a, b, c, u, v, w) e^{-i u} d c d u d v d w, a, b \in \mathbb{R},
$$

or even, as $\varphi_{1} \not \equiv 0$ and as $f$ may be changed by abelian translation,

$$
\int_{\mathbb{R}^{4}} f(a, b, c, u, v, w) c e^{-i u} d c d u d v d w=0, a, b \in \mathbb{R},
$$

i.e. $\widetilde{\Psi}_{1}(f)=0$. In particular, if $f$ is of the form $f=h_{1} \otimes \delta_{1}+h_{2} \otimes \delta_{2}$, then $h_{1}=0$, $f=h_{2} \otimes \delta_{2}$ and

$$
I^{\infty}=\left\langle h_{2} \otimes \delta_{2}\right\rangle+j\left(\pi_{l}\right)^{\infty} .
$$

Conversely, let us assume that $I^{\infty}=\left\langle h_{2} \otimes \delta_{2}\right\rangle+j\left(\pi_{l}\right)^{\infty}$ and let $\varphi \in I^{\perp}$ be of the form

$$
\varphi(a, b, c, u, v, w)=\left(\varphi_{2}(a, b) w+\varphi_{1}(a, b) c\right) e^{-i u}
$$

arbitrary. Then

$$
\int c f(a, b, c, u, v, w) e^{-i u} d c d u d v d w=h_{2}(a, b) \int_{N} p_{1}(n) \delta_{2}(n) \chi_{l}(n) d n=0
$$

and, by the computations of section 8.1.1,

$$
\begin{aligned}
0= & \int_{\mathbb{R}^{6}}\left(\varphi_{2}(a, b)(w-r c)+\varphi_{1}(a, b) c\right) \\
& \times e^{-i u} e^{-i(r b-s a)} h_{2}(a, b) \delta_{2}(c, u, v, w) d a d b d c d u d v d w \\
= & \int_{\mathbb{R}^{6}} \varphi_{2}(a, b) w e^{-i u} e^{-i(r b-s a)} h_{2}(a, b) \delta_{2}(c, u, v, w,) d a d b d c d u d v d w \\
= & \int_{\mathbb{R}^{2}} \varphi_{2}(a, b) h_{2}(a, b) e^{-i(r b-s a)} d a d b, r, s \in \mathbb{R} .
\end{aligned}
$$

As $I$ and $I^{\perp}$ are invariant by abelian translation and as $h_{2} \not \equiv 0$, the Fourier inversion theorem implies that $\varphi_{2} \equiv 0$ and that $\varphi$ is of the form $\varphi(a, b, c, u, v, w)=$ $\varphi_{1}(a, b) c e^{-i u}$.

Hence, in the first case, $I$ is the closure of $I^{\infty}=\left\langle h_{2} \otimes \delta_{2}\right\rangle+j\left(\pi_{l}\right)^{\infty}$ for some $0 \neq h_{2} \in \mathcal{S}\left(\mathbb{R}^{2}\right)$. It is then easy to check that $\left.\left(I^{\perp}\right)\right|_{N}=\left(\left.I\right|_{N}\right)^{\perp}=\left.\operatorname{span}\{1, c\} \cdot \chi_{l}\right|_{N}$. Hence $I$ is $L^{\infty}(G / N)$-invariant by Proposition 2.3 , a contradiction. So this first case does not occur.

8.1.3. Second case: Let us now assume that any $\varphi \in I^{\perp}$ of the form

$$
\varphi(a, b, c, u, v, w)=\left(\varphi_{2}(a, b) w+\varphi_{1}(a, b) c\right) e^{-i u}
$$

is such that $\varphi_{2} \not \equiv 0$. Hence there exists $\left(a_{0}, b_{0}\right) \in \mathbb{R}^{2}$ such that $\varphi_{2}\left(a_{0}, b_{0}\right) \neq 0$. Put

$$
\alpha:=\frac{\varphi_{1}\left(a_{0}, b_{0}\right)+i \partial_{2} \varphi_{2}\left(a_{0}, b_{0}\right)}{\varphi_{2}\left(a_{0}, b_{0}\right)} .
$$

By section 8.1.1, we have for all $f \in I^{S}$,

$$
0=\int_{\mathbb{R}^{4}}\left((w+\alpha c) f\left(a_{0}, b_{0}, c, u, v, w\right)+i c \partial_{2} f\left(a_{0}, b_{0}, c, u, v, w\right)\right) e^{-i u} d c d u d v d w
$$


As $f$ may be changed by arbitrary abelian translations (which commute with derivations), we obtain that

$$
0=\int_{\mathbb{R}^{4}}\left((w+\alpha c) f(a, b, c, u, v, w)+i c \partial_{2} f(a, b, c, u, v, w)\right) e^{-i u} d c d u d v d w,
$$

for all $a, b \in \mathbb{R}$. As translations in the directions of $N$ are also possible, this may be written as

$$
\widetilde{\Psi}_{2}(f)+\alpha \widetilde{\Psi}_{1}(f)+i \partial_{2} \widetilde{\Psi}_{1}(f)=0 .
$$

This means that for the given $I \in \mathcal{I}^{\pi_{\ell}}$, there exists $\alpha \in \mathbb{C}$ such that (8.12) is satisfied for all $f \in I$. In particular, for $f$ of the form $f=h_{1} \otimes \delta_{1}+h_{2} \otimes \delta_{2}$, this implies that $h_{2}=-\alpha h_{1}-i \partial_{2} h_{1}$. Moreover, (8.12) is satisfied identically by the elements of $j\left(\pi_{l}\right)^{\infty}$. If one also assumes that for $\tilde{\varphi} \in I^{\perp}$ of the form

$$
\tilde{\varphi}(a, b, c, u, v, w)=\left(\tilde{\varphi}_{2}(a, b) w+\tilde{\varphi}_{1}(a, b) c\right) e^{-i u},
$$

with $\tilde{\varphi}_{2}\left(a_{1}, b_{1}\right) \neq 0$ for some $\left(a_{1}, b_{1}\right) \in \mathbb{R}^{2}$, one may do the same reasoning as before for

$$
\beta=\frac{\tilde{\varphi}_{1}\left(a_{1}, b_{1}\right)+i \partial_{2} \tilde{\varphi}_{2}\left(a_{1}, b_{1}\right)}{\tilde{\varphi}_{2}\left(a_{1}, b_{1}\right)} .
$$

One then has

$$
\widetilde{\Psi}_{2}(f)+\beta \widetilde{\Psi}_{1}(f)+i \partial_{2} \widetilde{\Psi}_{1}(f)=0, \text { for all } f \in I^{S} .
$$

If $\alpha \neq \beta$, this implies that $\widetilde{\Psi}_{1}(f)=\widetilde{\Psi}_{2}(f)=0$, i.e.

$$
I^{S} \subset \operatorname{Ker} \widetilde{\Psi}_{1} \cap \operatorname{Ker} \widetilde{\Psi}_{2} \cap \operatorname{Ker} \pi_{l}=j\left(\pi_{l}\right) \cap \mathcal{S}(G),
$$

a contradiction. This shows that in the second case, $\alpha$ is uniquely determined and is valid for every $\varphi \in I^{\perp}$ of the given form.

It is easy to check that the set of solutions of (8.12) in $\operatorname{Ker} \pi_{l}$ is invariant by conjugation and by abelian translation. Hence its closure is invariant by left and right group translations, and it is an ideal $I_{\alpha}$ contained in $\operatorname{Ker} \pi_{l}$. In particular, if $f$ is a solution of (8.12), then the same is true for all $F \cdot f$, with $F \in \mathcal{S}(G \times G)$. By the definition of $\alpha$ and the previous arguments, $I$ is contained in one and only one ideal $I_{\alpha}$. Moreover, $I_{\alpha}$ is strictly contained in $\operatorname{Ker} \pi_{l}$. In fact, a function of the form $f=h_{1} \otimes \delta_{1}+h_{2} \otimes \delta_{2}$ with $h_{1}, h_{2} \in S\left(\mathbb{R}^{2}\right)$ such that $h_{2} \neq-\alpha h_{1}-i \partial_{2} h_{1}$ belongs to $\operatorname{Ker} \pi_{l}$, but not to $I_{\alpha}$. For any $h \in \mathcal{S}\left(\mathbb{R}^{2}\right)$, let us define

$$
f_{h, \alpha}=h \otimes \delta_{1}+\left(-\alpha h-i \partial_{2} h\right) \otimes \delta_{2} .
$$

We have shown that there exists exactly one $\alpha \in \mathbb{C}$ and a function $h \in \mathcal{S}\left(\mathbb{R}^{2}\right)$ such that

$$
I^{\infty}=\left\langle f_{h, \alpha}\right\rangle+j\left(\pi_{l}\right)^{\infty} .
$$

We will now show that the function $0 \neq h \in \mathcal{S}\left(\mathbb{R}^{2}\right)$ in 8.13 may be chosen arbitrarily and that $I=I_{\alpha}$. In order to use the results of Remark 4.2 and Proposition 4.4. we first have to replace the elements $\widetilde{\Psi}_{j}(f)$ of $\mathcal{S}\left(G / N, \chi_{l}\right)$ by the $\Psi_{j}(f)$ 's defined in section 4 by

$$
\Psi_{j}(f)(x):=\int_{N} f(x n) p_{j}(n) d n, j=1,2,
$$


for all $f \in \mathcal{S}(G)$. An easy computation shows that

$$
\begin{aligned}
\widetilde{\Psi}_{1}(f) & =\Psi_{1}(f), \\
\widetilde{\Psi}_{2}(f)(a, b, c, u, v, w) & =\Psi_{2}(f)(a, b, c, u, v, w)-\frac{1}{2} a \Psi_{1}(f)(a, b, c, u, v, w),
\end{aligned}
$$

for all $(a, b, c, u, v, w) \in G \equiv \mathbb{R}^{6}$ and equation (8.12) is equivalent to

$$
\begin{aligned}
& \Psi_{2}(f)(a, b, c, u, v, w)-\frac{1}{2} a \Psi_{1}(f)(a, b, c, u, v, w)+\alpha \Psi_{1}(f)(a, b, c, u, v, w) \\
& +i \partial_{2} \Psi_{1}(f)(a, b, c, u, v, w)=0,
\end{aligned}
$$

for all $(a, b, c, u, v, w) \in \mathbb{R}^{6}$. In particular,

$$
\operatorname{Ker} \Psi_{1} \cap \operatorname{Ker} \Psi_{2} \cap \operatorname{Ker} \pi_{l}=\operatorname{Ker} \widetilde{\Psi}_{1} \cap \operatorname{Ker} \widetilde{\Psi}_{2} \cap \operatorname{Ker} \pi_{l}=j\left(\pi_{l}\right) \cap \mathcal{S}(G) .
$$

In order to use Proposition 4.4, let us recall that a Jordan-Hölder series for the action of $G \times N$ on $\mathcal{P}_{\left.\omega\right|_{N}}(N)$ is given by

$$
W_{0}=\mathbb{C} \subset W_{1}=\operatorname{span}\left\{1, p_{1}\right\} \subset W_{2}=\operatorname{span}\left\{1, p_{1}, p_{2}\right\}=\mathcal{P}_{\left.\omega\right|_{N}}(N),
$$

and hence

$$
j\left(\pi_{l}\right)=I_{W_{2}} \subset I_{W_{1}} \subset I_{W_{0}}=\operatorname{Ker} \pi_{l} .
$$

Let us notice that for $f, g \in \operatorname{Ker} \pi_{l} \cap \mathcal{S}(G)$ arbitrary with $\Psi_{1}(f) \neq 0$, there exists $F \in \mathcal{S}(G \times G)$ such that

$$
\Psi_{1}(g)=F \cdot \Psi_{1}(f)=\Psi_{1}(F \cdot f),
$$

by Remark 4.2 and Proposition 4.4. Hence $F \cdot f-g \in \operatorname{Ker} \Psi_{1}$. If, moreover, $f, g \in I_{\alpha}$, i.e. $f, g$ satisfy (8.12), then

$$
g-F \cdot f \in \operatorname{Ker} \Psi_{1} \cap \operatorname{Ker} \Psi_{2} \cap \operatorname{Ker} \pi_{l}=j\left(\pi_{l}\right) \cap \mathcal{S}(G) .
$$

This shows that

$$
\langle g\rangle+j\left(\pi_{l}\right)^{\infty} \subset\langle f\rangle+j\left(\pi_{l}\right)^{\infty}
$$

and conversely, by exchanging the roles of $f$ and $g$. Hence

$$
I=\left\langle f_{h, \alpha}\right\rangle+j\left(\pi_{l}\right)^{\infty}
$$

for any $0 \neq h \in \mathcal{S}\left(\mathbb{R}^{2}\right)$. The same procedure may be applied to the ideal $I_{\alpha}$, which is then shown to be of the same type as $I$. Hence $I=I_{\alpha}$.

We then have the following proposition:

Proposition 8.2. Let $V=\mathcal{P}_{\left.\omega\right|_{N}}(N)$ and $W=\mathbb{C}$. There exists for every $\alpha \in \mathbb{C} a$ unique closed two-sided ideal $I_{\alpha} \in \mathcal{I}^{\pi_{\ell}}$ such that

$$
I_{\alpha} \neq I_{\beta} \text { if } \alpha \neq \beta \text { and } j\left(\pi_{\ell}\right)=I_{V} \subsetneq I_{\alpha} \subsetneq I_{W}=k e r\left(\pi_{\ell}\right) .
$$

These ideals $I_{\alpha}$ are not $L^{\infty}(G / N)$-invariant. Furthermore, for every ideal $I \in \mathcal{I}^{\pi_{\ell}}$ which is not $L^{\infty}(G / N)$-invariant, there exists a unique $\alpha \in \mathbb{C}$ such that $I=I_{\alpha}$. The ideal $I_{\alpha}$ is the closure of

$$
I_{\alpha}^{\infty}=\left\langle f_{h, \alpha}\right\rangle+j\left(\pi_{l}\right)^{\infty}
$$

for any $0 \neq h \in \mathcal{S}\left(\mathbb{R}^{2}\right)$, where $f_{h, \alpha}=h \otimes \delta_{1}+\left(-\alpha h-i \partial_{2} h\right) \otimes \delta_{2}$. 
8.3. The threadlike case. Let $\mathfrak{g}=\operatorname{fad}_{4}, \mathfrak{g}=\operatorname{span}\{T, X, Y, Z\}$ with the brackets

$$
[T, X]=Y,[T, Y]=Z \text {. }
$$

On the group $\mathrm{Fad}_{4}$ we take the coordinates

$$
(t, x, y, z) \equiv \exp (t T) \exp (x X) \exp (y Y+z Z) .
$$

Let $\ell \in \mathfrak{g}^{*}, \ell(Y)=1, \ell(Z)=\ell(T)=\ell(X)=0$. Then $\mathfrak{g}(\ell)=\mathfrak{n}=\operatorname{span}\{Y, Z\}$.

8.3.1. Degree one weight. Let $\omega$ be a polynomial weight on $F a d_{4}$, such that $\left.\omega\right|_{N}$ is a polynomial weight of degree 1 on $N$. One may for instance take $\omega(t, x, y, z)=$ $1+|t|+|x|+|y|+|z|+\frac{1}{2} t^{2}$, which gives $\left.\omega\right|_{N}(0,0, y, z)=1+|y|+|z|$.

Let $I \in \mathcal{I}^{\pi_{\ell}}$. Suppose that $W(I)=\mathbb{C}, V(I)=\operatorname{span}\{1, z, y\}=\mathcal{P}_{\left.\omega\right|_{N}}(N)$. This gives us the Jordan-Hölder basis $\left\{p_{1}=y, p_{2}=z\right\}$ of the $G \times N$-module $V(I) / W(I)$ and $\iota^{I}=\{1\}$. As $I \subset \operatorname{Ker} \pi_{l}$, we have for all $f \in I^{S}$, for all $t, x \in \mathbb{R}$, $\left.\exp (-t T) f_{\exp (x X)}\right|_{N} \in \operatorname{Ker} \chi_{\left.l\right|_{\mathfrak{n}}}$, and hence

$$
\int_{\mathbb{R}^{2}} f(t, x, y, z) e^{-i y} d y d z=0, t, x \in \mathbb{R}
$$

Let $\varphi \in\left(I^{\perp}\right)^{\infty}$ and let us write

$$
\begin{array}{rcl}
\varphi(t, x, y, z) & =\quad \chi_{l}(t, x, y, z) \psi(t, x, y, z) \\
\text { where } & \psi(t, x, y, z)=\psi_{0}(t, x) z+\psi_{1}(t, x) y \\
\text { and } & \chi_{l}(t, x, y, z)=e^{-i y}, t, x, y, z \in \mathbb{R} .
\end{array}
$$

As in the step 2 case, we don't need to consider any possible term of the form $\psi_{00}(t, x) \cdot \chi_{l}(t, x, y, z)$ in the expression of $\varphi$, since $I \subset \operatorname{ker}\left(\pi_{l}\right)=e_{G}\left(\operatorname{ker}\left(\chi_{l}\right)\right)$. After conjugating $\varphi$ with $\exp (s X)$ we get, by Fourier inversion,

$$
0=\int_{\mathbb{R}^{3}} \varphi(t, x, y, z) f(t, x, y, z) d x d y d z, f \in I^{S}, t \in \mathbb{R},
$$

and conjugating $\varphi$ with $\exp (s T)$ we get the identity

$$
0=\int_{\mathbb{R}^{3}} \varphi\left(t, x, y+s x, z+s y+\frac{s^{2}}{2} x\right) f(t, x, y, z) d x d y d z, f \in I^{S}, t, s \in \mathbb{R} .
$$

Hence, for $t=0$, relation (8.14) implies that

$0=\int_{\mathbb{R}^{3}}\left(\psi_{0}(0, x) z+\left(\psi_{1}(0, x)+s \psi_{0}(0, x) y\right)\right) f(0, x, y, z) e^{-i y} e^{-i s x} d x d y d z, s \in \mathbb{R}, f \in I^{S}$.

Finally, integration by parts and a Fourier inversion theorem give, at $x=0$,

$$
\begin{aligned}
0= & \int_{\mathbb{R}^{2}}\left(\psi_{0}(0,0) z+\left(\psi_{1}(0,0)-i \partial_{x} \psi_{0}(0,0) y\right)\right) f(0,0, y, z) e^{-i y} d y d z \\
& -i \int_{\mathbb{R}^{2}} \psi_{0}(0,0) y \partial_{x} f(0,0, y, z) e^{-i y} d y d s, f \in I^{S} .
\end{aligned}
$$


If we replace $f$ by its right translates with $\exp (x X)$ and its left translates with $\exp (t T)$, we finally have that

$$
\begin{aligned}
0= & \psi_{0}(0,0) \int_{\mathbb{R}^{2}} z e^{-i y} f(t, x, y, z) d y d z \\
& +\left(\psi_{1}(0,0)-i \partial_{x} \psi_{0}(0,0)\right) \int_{\mathbb{R}^{2}} y e^{-i y} f(t, x, y, z) d y d z \\
& -i \psi_{0}(0,0) \int_{\mathbb{R}^{2}} y e^{-i y} \partial_{x} f(t, x, y, z) d y d z, f \in I^{S}, t, x \in \mathbb{R} .
\end{aligned}
$$

We now have to distinguish two cases:

First case. For all such $\varphi$ 's and $\psi$ 's, $\psi_{0} \equiv 0$, i.e. all such $\varphi$ 's are of the form

$$
\varphi(t, x, y, z)=\psi_{1}(t, x) y e^{-i y} \text {. }
$$

We proceed as in the previous example (section 8.1.2) and conclude that in this case the ideal $I$ is $L^{\infty}(G / N)$-invariant.

Second case. Let us assume that there exist $\varphi, \psi$ such that $\psi_{0} \not \equiv 0$. As we may translate to the left by $\exp (t T)$ and to the right by $\exp (x X)$, we may assume that $\psi_{0}(0,0) \neq 0$. Let us then put

$$
\alpha=\frac{\psi_{1}(0,0)-i \partial_{x} \psi_{0}(0,0)}{\psi_{0}(0,0)}
$$

As in section 8.1.3, we define $\Psi_{j}(f)$ by

$$
\Psi_{j}(f)(x):=\int_{N} f(x n) p_{j}(n) d n, j=1,2 .
$$

Because $f$ may be translated to the right by any element of $N$, equation (8.15) may be written as

$$
\Psi_{2}(f)+\alpha \Psi_{1}(f)-i \partial_{x} \Psi_{1}(f)=0, f \in I^{S},
$$

with $\partial_{x} \Psi_{1}(f)=\Psi_{1}\left(\partial_{x} f\right)$. We may then conclude as in the step two case (with the simplification that we don't need to distinguish between $\Psi_{j}$ and $\widetilde{\Psi}_{j}$ ):

Proposition 8.4. There exists for every $\alpha \in \mathbb{C}$ exactly one ideal $I_{\alpha} \in \mathcal{I}^{\pi_{\ell}}$ which is a solution to equation (8.15) and which is not $L^{\infty}(G / N)$-invariant. All the ideals $I \in \mathcal{I}^{\pi_{\ell}}$ which are not $L^{\infty}(G / N)$-invariant are of that form.

8.4.1. Degree two weight. As before let $\ell \in \mathfrak{g}^{*}$ with $\ell(Y)=1, \ell(Z)=\ell(T)=\ell(X)=$ 0 . Let $\omega$ be a polynomial weight on $\mathrm{Fad}_{4}$ such that $\mathcal{P}_{\omega_{\mid N}}(N)$ admits polynomials of degree $\leq 2$. This case is far more complex, and we will thus limit ourselves to proving the existence of one family of ideals, indexed by $\mathbb{C}^{2}$, contained in $\mathcal{I}^{\pi_{\ell}}$ and which are not $L^{\infty}(G / N)$-invariant.

Let $W=\mathbb{C}+\operatorname{span}\{y, z\}$ and $V=\operatorname{span}\left\{z^{2}, z y, y^{2}, z, y, 1\right\}$. We will show in the following that there exist ideals $I \in \mathcal{I}^{\pi_{\ell}}$ such that $W(I)=W$ and $V(I)=V$. Let us assume that such $I$ 's exist. We have the Jordan-Hölder basis $\left\{p_{1}=y^{2}, p_{2}=\right.$ $\left.y z, p_{3}=z^{2}\right\}$ of $V(I) / W(I)$. We assume that $I$ is not $L^{\infty}(G / N)$-invariant. Hence $I_{V(I)} \subsetneq I \subsetneq I_{W(I)}$ and $\left.I\right|_{N}$ is annihilated by $\operatorname{span}\{1, y, z\} \cdot \chi_{\left.l\right|_{\mathfrak{n}}}$. 
It is therefore enough to consider $\varphi^{\prime}$ s in $I^{\perp}$ of the following form:

$$
\begin{array}{ccl}
\varphi(t, x, y, z) & =\quad(t, x, y, z) \psi(t, x, y, z) \\
\text { where } & \psi(t, x, y, z)=\psi_{0}(t, x) z^{2}+\psi_{1}(t, x) z y+\psi_{2}(t, x) y^{2} \\
\text { and } & \chi(t, x, y, z)=e^{-i y}, t, x, y, z \in \mathbb{R} .
\end{array}
$$

Suppose that $\psi_{0} \not \equiv 0$. As we may translate to the left by $\exp (t T)$ and to the right by $\exp (x X)$, we may assume that $\psi_{0}(0,0) \neq 0$, and even that $\psi_{0}(0,0)=1$.

By conjugating $\varphi$ with $\exp (s X)$ and using the previous remark about the annihilation of elements of $\left.I\right|_{N}$, we see that

$$
0=\int_{\mathbb{R}^{3}} \varphi(t, x, y, z) f(t, x, y, z) d x d y d z, f \in I^{S}, t \in \mathbb{R} .
$$

By conjugating $\varphi$ with $\exp (s T)$ and using the same remark about annihilation, we obtain the identity

$$
0=\int_{\mathbb{R}^{3}} \varphi\left(t, x, y+s x, z+s y+\frac{s^{2}}{2} x\right) f(t, x, y, z) d x d y d z, f \in I^{S}, t \in \mathbb{R} .
$$

Hence, for $t=0$,

$$
\begin{aligned}
0= & \int_{\mathbb{R}^{3}} f(0, x, y, z)\left(\psi_{0}(0, x)\left(z+s y+\frac{s^{2}}{2} x\right)^{2}+\psi_{1}(0, x)\left(z+s y+\frac{s^{2}}{2} x\right) y\right. \\
& \left.+\psi_{2}(0, x) y^{2}\right) e^{-i y-i s x} d x d y d z \\
= & \int_{\mathbb{R}^{3}} f(0, x, y, z)\left(\psi_{0}(0, x)(z+s y)^{2}+\psi_{1}(0, x)(z+s y) y+\psi_{2}(0, x) y^{2} e^{-i y-i s x} d x d y d z\right. \\
= & \int_{\mathbb{R}^{3}} f(0, x, y, z)\left(\psi_{0}(0, x) z^{2}+\left(2 s \psi_{0}(0, x)+\psi_{1}(0, x)\right) z y\right. \\
& \left.+\left(s^{2} \psi_{0}(0, x)+s \psi_{1}(0, x)+\psi_{2}(0, x)\right) y^{2}\right) e^{-i y-i s x} d x d y d z, s \in \mathbb{R}, f \in I^{S} .
\end{aligned}
$$

Therefore we get the equation

$$
\begin{aligned}
0= & \int_{\mathbb{R}^{2}} f(0, x, y, z)\left(\psi_{0}(0, x) z^{2}+\psi_{1}(0, x) z y+\psi_{2}(0, x) y^{2} e^{-i y} d y d z\right. \\
& -2 i \int_{\mathbb{R}^{2}} \partial_{x}(f(0, x, y, z)) \psi_{0}(0, x) z y e^{-i y} d y d z \\
& -2 i \int_{\mathbb{R}^{2}} f(0, x, y, z) \partial_{x} \psi_{0}(0, x) z y e^{-i y} d y d z \\
& -i \int_{\mathbb{R}^{2}} \partial_{x} f(0, x, y, z) \psi_{1}(0, x) y^{2} e^{-i y} d y d z \\
& -i \int_{\mathbb{R}^{2}} f(0, x, y, z) \partial_{x} \psi_{1}(0, x) y^{2} e^{-i y} d y d z \\
& -\int_{\mathbb{R}^{2}} \partial_{x}^{2} f(0, x, y, z) \psi_{0}(0, x) y^{2} e^{-i y} d y d z \\
& -2 \int_{\mathbb{R}^{2}} \partial_{x} f(0, x, y, z) \partial_{x} \psi_{0}(0, x) y^{2} e^{-i y} d y d z \\
& -\int_{\mathbb{R}^{2}} f(0, x, y, z) \partial_{x}^{2} \psi_{0}(0, x) y^{2} e^{-i y} d y d z, f \in I^{S} .
\end{aligned}
$$


Hence, putting first $t=x=0$ and then, in a second step, translating $f$ again by $\exp (t T)$ on the left and by $\exp (x X)$ on the right, we get

$$
\begin{aligned}
0= & \int_{\mathbb{R}^{2}} f(t, x, y, z) z^{2} e^{-i y} d y d z \\
& +\left(\psi_{1}(0,0)-2 i \partial_{x} \psi_{0}(0,0)\right) \int_{\mathbb{R}^{2}} f(t, x, y, z) z y e^{-i y} d y d z \\
& +\left(\psi_{2}(0,0)-i \partial_{x} \psi_{1}(0,0)-\partial_{x}^{2} \psi_{0}(0,0)\right) \int_{\mathbb{R}^{2}} f(t, x, y, z) y^{2} e^{-i y} d y d z \\
& -2 i \int_{\mathbb{R}^{2}} \partial_{x} f(t, x, y, z) z y e^{-i y} d y d z \\
& -i\left(-2 i \partial_{x} \psi_{0}(0,0)+\psi_{1}(0,0)\right) \int_{\mathbb{R}^{2}} \partial_{x} f(t, x, y, z) y^{2} e^{-i y} d y d z \\
& -\int_{\mathbb{R}^{2}} \partial_{x}^{2} f(t, x, y, z) y^{2} e^{-i y} d y d z, f \in I^{S} .
\end{aligned}
$$

As, moreover, $f$ may be translated to the right by any element of $N$, this gives us for $f \in I^{S}$ the relations

$$
\Psi_{3}(f)+a_{2} \Psi_{2}(f)+a_{1} \Psi_{1}(f)-2 i \Psi_{2}\left(\partial_{x} f\right)-\Psi_{1}\left(\partial_{x}^{2} f\right)-i a_{2} \Psi_{1}\left(\partial_{x} f\right)=0
$$

where the constants $a_{1}, a_{2} \in \mathbb{C}$ are obtained by $a_{2}=\psi_{1}(0,0)-2 i \partial_{x} \psi_{0}(0,0), a_{1}=$ $\psi_{2}(0,0)-i \partial_{x} \psi_{1}(0,0)-\partial_{x}^{2} \psi_{0}(0,0)$. The functions $\Psi_{j}$ 's are defined as in section 8.1 . By definition of the $\Psi_{j}$ 's,

$$
\operatorname{Ker} \Psi_{1} \cap \operatorname{Ker} \Psi_{2} \cap \operatorname{Ker} \Psi_{3} \cap I_{W(I)} \cap \mathcal{S}(G)=I_{V(I)} \cap \mathcal{S}(G) .
$$

Now choose $f=\sum_{j=1}^{3} h_{j} \otimes \delta_{j} \in I_{S}$, where as before $\delta_{j} \in \mathcal{S}(N)$ and

$$
\begin{gathered}
0=\int_{N} \delta_{j}(n) \chi_{\ell}(n) d n, \int_{N} \delta_{k}(n) q_{k}(n) \chi_{l}(n) d n=0, \\
\int_{N} \delta_{j}(n) p_{i}(n) \chi_{\ell}(n) d n=\delta_{i j}, 1 \leq i, j \leq 3,1 \leq k \leq 2,
\end{gathered}
$$

for $q_{1}(y, z)=y, q_{2}(y, z)=z$. Then equation (8.18) tells us that

$$
h_{3}+a_{2} h_{2}+a_{1} h_{1}-2 i \partial_{x} h_{2}-\partial_{x}^{2} h_{1}-i a_{2} \partial_{x} h_{1}=0 .
$$

Conversely, two independent solutions of equation (8.18) may be obtained in the following way: Choose a function $h \neq 0$ in $\mathcal{S}(\mathbb{R})$ and let

$$
f_{1}=h \otimes \delta_{1}-\left(a_{1} h-\partial_{x}^{2} h-i a_{2} \partial_{x} h\right) \otimes \delta_{3}, f_{2}=h \otimes \delta_{2}-\left(a_{2} h-2 i \partial_{x} h\right) \otimes \delta_{3} .
$$

We obtain the ideal $I_{a_{1}, a_{2}}$ as closure of the space

$$
I_{a_{1}, a_{2}}^{\infty}=\left\langle f_{1}, f_{2}\right\rangle+I_{V(I)}^{\infty},
$$

where $V(I)=\operatorname{span}\left\{1, y, z, y^{2}, y z, z^{2}\right\}$. For this ideal we have $\iota^{I_{a_{1}, a_{2}}}=\{1,2\}$ and $I_{V(I)} \subsetneq I_{a_{1}, a_{2}} \subsetneq I_{W(I)}$. In particular, $I_{a_{1}, a_{2}} \in \mathcal{I}^{\pi_{\ell}}$ and it is not $L^{\infty}(G / N)$-invariant. We hence have:

Proposition 8.5. For every $\left(a_{1}, a_{2}\right) \in \mathbb{C}^{2}$, there exists an ideal $I_{a_{1}, a_{2}} \in \mathcal{I}^{\pi_{\ell}}$, which is not $L^{\infty}(G / N)$-invariant. The elements $f$ of $I_{a_{1}, a_{2}}^{\infty}$ satisfy

$$
\Psi_{3}(f)+a_{2} \Psi_{2}(F)+a_{1} \Psi_{1}(f)-2 i \Psi_{2}\left(\partial_{x} f\right)-\Psi_{1}\left(\partial_{x}^{2} f\right)-i a_{2} \Psi_{1}\left(\partial_{x} f\right)=0 .
$$




\section{REFERENCES}

[Al-Lu] D. Alexander and J. Ludwig, Minimal ideals of group algebras, Studia Math. 160, no. 3 (2004), 205-229. MR2033400 (2004k:43007)

[Co-Gr] L. Corwin and P. Greenleaf, Representations of nilpotent Lie groups and their applications. Part 1: Basic theory and examples, Cambridge Studies in Advanced Mathematics, 18 (1990). MR:1070979 (92b:22007)

[Di] J. Dixmier, Opérateurs de rang fini dans les représentations unitaires, Publ. Math. IHES 6 (1960), 305-317. MR0136684 (25:149)

[Dz-Lu-Mo] J. Dziubanski, J. Ludwig and C. Molitor-Braun, Functional Calculus in Weighted Group Algebras, Rev. Mat. Complut. 17, Núm. 2 (2004), 321-357. MR2083958 (2005f:43003)

[Ho] R. Howe, On a connection between nilpotent groups and oscillatory integrals associated to singularities, Pacific J. Math. 73 (1977), no. 2, 329-363. MR0578891 $(58: 28270)$

[Le-Lu] H. Leptin, J. Ludwig, Unitary representation theory of exponential Lie groups, De Gruyter Expositions in Mathematics 18, 1994. MR1307383 (96e:22001)

[Lue-Po] A. Lüdeking, D. Poguntke, Cocycles on abelian groups and primitive ideals in group $C^{*}$-algebras of two-step nilpotent groups and connected Lie groups, Journal of Lie Theory 4 (1994), 39-103. MR1326951 (96i:22013)

[Lu] J. Ludwig, Good Ideals in the Group Algebra of a Nilpotent Lie Group, Mathematische Zeitschrift 161 (1978), 195-210. MR0498970 (58:16958)

[Lu1] J. Ludwig, Polynomial growth and ideals in group algebras, Manuscripta Math. 30 (1980), no. 3, 215-221. MR.557105 (81e:43012)

[Lu2] J. Ludwig, On primary ideals in the group algebra of a nilpotent Lie group. Math. Ann., 262 (1983), 287-304. MR692858 (84h:22016)

[Lu3] J. Ludwig, On the Hilbert- Schmidt seminorms of $L^{1}$ of a nilpotent Lie group, Math. Ann. 273 (1986), no. 3, 383-395. MR824429 (87e:22018)

[Lu4] J. Ludwig, Minimal $C^{*}$-Dense Ideals and Algebraically Irreducible Representations of the Schwartz-Algebra of a Nilpotent Lie group, Harmonic Analysis, Proceedings, Luxembourg 1987, Lecture Notes in Math. 1359, Springer. MR974317 (90c:22019)

[Lu-Mo] J. Ludwig and C. Molitor-Braun, Algèbre de Schwartz d'un groupe de Lie nilpotent, Travaux mathématiques VII, Publications du Centre Universitaire de Luxembourg (1995), 25-67.

[Lu-Mo-1] J. Ludwig and C. Molitor-Braun, Représentations irréductibles bornées des groupes de Lie exponentiels, Canad. J. Math. 53 (5) (2001), 944-978. MR.1859763 (2002h:22015)

[Lu-Mo-2] J. Ludwig and C. Molitor-Braun, Flat orbits, minimal ideals and spectral synthesis, Monatshefte Math. 160 (2010), 271-312. MR2661313(2011f:22013)

[Po] D. Poguntke, Algebraically irreducible representations of $L^{1}$-algebras of exponential groups, Duke Math. J. 50 (4), (1983), 1077-1106. MR726318(85e:22014)

Laboratoire LMAM, UMR 7122, Université de Lorraine, Ile de Saulcy, F-57045 Metz CEDEX 1, FRANCE

E-mail address: jean.ludwig@univ-lorraine.fr

Unité de Recherche en Mathématiques, Université du Luxembourg, 6, Rue CoudenhoveKAlergi, L-1359 Luxembourg, Luxembourg

E-mail address: carine.molitor@uni.lu

Fakultät für Mathematik, Universität Bielefeld, Postfach 100131, D-33501 BieleFELD, GERMANY

E-mail address: poguntke@math.uni-bielefeld.de 